\title{
A theoretical study of porphyrin isomers and their core-modified analogues: cis-trans isomerism, tautomerism and relative stabilities
}

\author{
M PUNNAGAI, SAJU JOSEPH and G NARAHARI SASTRY* \\ Molecular Modelling Group, Organic Chemical Sciences, Indian Institute of Chemical Technology, \\ Hyderabad 500007 India \\ e-mail: gnsastry@iict.res.in; gnsastry@yahoo.com
}

MS received 4 May 2004; revised 11 August 2004

\begin{abstract}
Semiempirical (AM1 and PM3) and density functional theory (DFT) calculations were performed on about 50 porphyrin isomers with 25 each of 1,2 (syn) and 1,3 (anti) tautomeric forms. The corresponding oxa- and thia-core-modified analogues were also computed. The variations of relative energies and stabilities of the core-modified analogues were compared with parent porphyrin 1 and the corresponding oxa- and thia-analogues. The trends in relative energies are not significantly changed while going from parent system to oxa- and thia-core-modified porphyrins in case of both syn and anti

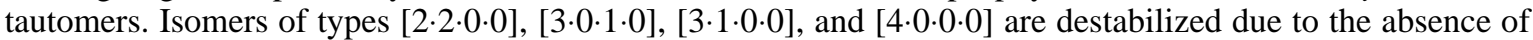

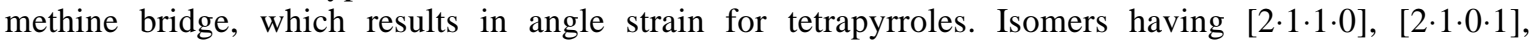
$[2 \cdot 0 \cdot 2 \cdot 0]$ and $[2 \cdot 2 \cdot 0 \cdot 0]$ connectivity, the $Z$ isomers, are more stable compared to the corresponding $E$ isomers in both syn and anti forms of parent and core-modified analogues.
\end{abstract}

Keywords. Porphyrin isomers; core-modified analogues; theoretical studies; tautomerism; oxa-porphyrin; thiaporphyrin.

\section{Introduction}

Study of porphyrins has developed into a subject of interdisciplinary research due to their importance and relevance in a wide range of areas such as chemistry, biology and medicine. ${ }^{1-3}$ These highly coloured tetrapyrrolic macrocyclic pigments ${ }^{4}$ play a diverse and critical role in biology, ranging from electron transfer, oxygen transport and storage, and photosynthetic processes to catalytic substrate oxidation. ${ }^{2,3}$ Recently there have been a number of reports on the use of modified, expanded and isomeric forms of porphyrins as molecular receptors for various substrates. ${ }^{5}$ Other tetrapyrrolic structures, such as chlorins, phthallocyanines, porphyrin-ketones and benzoporphyrins have shown interesting phosphorescence properties. $^{6}$

Theoretical research on porphyrins, like other aspects of porphyrin chemistry, is also driven by considerations of great biological importance of these molecules, their extensive coordination chemistry,

\footnotetext{
*For correspondence

'IICT Communication No. 040404
}

and growing number of applications. ${ }^{1-7}$ Thus, porphyrin-like molecules, structural variants of tetrapyrrolic macrocycles possessing $(4 n+2) \pi$ electron delocalization, have been the favourite hunting ground for synthetic chemists in anticipation of their special properties. Therefore, there is a major thrust in designing and synthesizing structural variants of basic tetrapyrrole macro cycles in contemporary research. ${ }^{5}$ Research on the synthesis of porphyrin isomers has gained momentum since the discovery of porphycene by Vogel and co-workers and $\mathrm{N}$-confused porphyrin independently by Furuta et al and LatosGrazynski and co-workers. ${ }^{8}$ The porphyrin isomers reported to date include, corrphycene, hemiporphycene, and isoporphycene obtained by shuffling the four pyrrolic subunits and the meso-carbon bridge. ${ }^{5,8-12}$ Many research groups have recently exploited some of the unique properties exhibited by the N-confused porphyrins because their remarkable ability to act as tetra-coordinate ligands to form transition metal complexes. ${ }^{4}$ This led to a flurry of synthetic attempts toward novel porphyrin-like molecules, with structural variants such as ring or bridge extended, reshuffled, inverted, $\mathrm{N}$ - and $\mathrm{C}$-fused, contracted and core-modified porphyrins..$^{5,11-13}$ 
Wu et al carried out density functional calculations using both the BLYP/3-21G and BLYP/6$31 \mathrm{G}^{* *}$ methods on free-base porphyrins and their possible isomers with an $\mathrm{N}_{4}$-metal coordination core. ${ }^{9}$ Ghosh and Jynge reported BLYP/6-31G** calculations on cis and trans porphyrin isomers having $[3 \cdot 0 \cdot 1 \cdot 0],[3 \cdot 1 \cdot 0 \cdot 0]$ and $[4 \cdot 0 \cdot 0 \cdot 0]$ connectivity. ${ }^{10}$ Core modification in the skeleton by replacing one or two pyrrole nitrogens by other heteroatoms such as $\mathrm{O}$ and $\mathrm{S}$ alters the electronic structure and results in the formation of modified porphyrins. Introducing other heteroatoms yields new classes of compounds containing novel chelating properties.

The present study is aimed at tetrapyrrolic porphyrin isomers with $\mathrm{N}_{4}$ cores and their corresponding core-modified structures where the two NH groups are replaced by oxygen and sulphur as heteroatoms. Two types of tautomers, (a) the imino protons on III and I ring (anti type), scheme 1; (b) imino protons at II and I ring (syn type), scheme 2 are taken into consideration. Thus a total of 150 isomers are considered here. The isomers of the parent porphyrin are divided into eight classes based on the nature of bridging, viz. freebase porphyrin $[1 \cdot 1 \cdot 1 \cdot 1]$, hemiporphycene $[2 \cdot 1 \cdot 1 \cdot 0]$, corrphycene $[2 \cdot 1 \cdot 0 \cdot 1]$, porphycene $[2 \cdot 0 \cdot 2 \cdot 0]$, isoporphycene $[3 \cdot 0 \cdot 1 \cdot 0],[2 \cdot 2 \cdot 0 \cdot 0]$, [3.1.0.0] and [4.0.0.0]. Schemes 1 and 2 depict all the structures considered in the study along with the nomenclature. Except for the freebase 1, all other classes involving geometrical isomerism $Z(\mathrm{cis})$ and $E$ (trans) in both syn and anti tautomeric forms are studied.

\section{Computational methods}

All the structures considered in the study (schemes 1 and 2) were initially taken as planar, and the geometry optimisations and frequency calculations were done to characterize whether they are minima on the potential energy surface or not. AM1 frequency calculations on the planar forms of most isomers indicate that none of them are minima on the potential energy surface. Subsequently, all the non-planar stationary points were found without imposing any symmetry constraints and characterized as minima with all real frequencies. Considering the size and the number of compounds involved in the study, doing full geometry optimisations at higher levels of theory was too expensive. Therefore, we restricted ourselves to single point calculations at B3LYP/6$31 \mathrm{G}^{*}$ level on AM1 optimised geometries. All the structures are optimised at the semiempirical AM $1^{14}$ and PM $3{ }^{15}$ methods. All the calculations in the study are performed using the Gaussian 98 suite of programs. ${ }^{16}$ The relative energies of all the isomers are computed considering the parent porphyrin $\mathbf{1}$ and the corresponding oxa- and thiaanalogues. The distortion energy for each isomer is computed by taking the difference between the planar structure and the corresponding minimum energy structure.

\section{Results and discussion}

Different types of isomers are arrived at by varying the methine bridges in addition to the parent porphyrin moiety. Considering all the possible geometric isomers, totally 25 structures in each of 1,3 (anti) and 1,2 (syn) tautomers are considered in the present study, as given in schemes 1 and 2. First we discuss the relative energies and structural preferences in the anti-isomers of the parent and its core-modified analogues. This is followed by a discussion on the corresponding syn tautomeric forms. Finally, the geometric preference between the $E$ and $Z$ isomers in both syn and anti forms is discussed. The relative energies calculated for the porphyrin isomers at AM1, PM3 and B3LYP levels of theory are given in tables 1-6. While the main purpose of the study is to examine the relative energy variations in porphyrin and related isomers, the current study also addresses the applicability of semiempirical methodologies to this type of systems.

\subsection{Anti tautomeric form}

The relative energies and distortion energies of the anti tautomers of parent porphyrin and core-modified porphyrin analogues are given in tables 1-3. Among the 25 distinct isomers considered, $3 \mathbf{c}-\mathrm{NH}$, $4 \mathbf{e}-\mathrm{NH}$, and $\mathbf{5 f}-\mathrm{NH}$ upon unrestrained geometry optimisation have collapsed to the closely related isomers 3b-NH, 4d-NH, and 5d-NH respectively. Thus, the total number of distinct minimum energy isomers of the porphyrin isomers has reduced to 22 . Exactly the same trends were witnessed even for the oxy-modified analogues. However, among the thiaanalogues, the minimum energy structures of $\mathbf{4 d}$ $\mathrm{S}$ and $\mathbf{4 e - S}$ have distinct geometries, while $\mathbf{2 c - S}$ and $\mathbf{2 b}-\mathbf{S}$ and isomers $\mathbf{3 c}-\mathrm{S}$ and $\mathbf{3 b}-\mathrm{S}$ collapse to an identical geometry after optimisation. In this section, we discuss the anti tautomeric structures and the results of the parent system are compared of with those of 


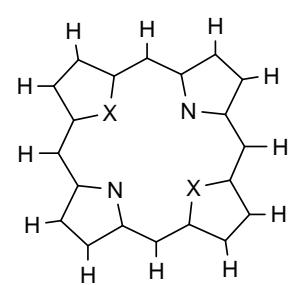

$1-\mathrm{X} ;[1 \cdot 1 \cdot 1 \cdot 1]$

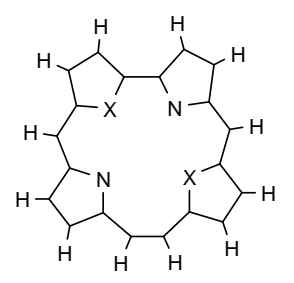

3a-X; $[2 \cdot 1 \cdot 0 \cdot 1]$

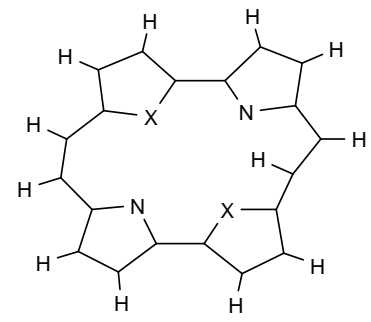

4b-X; [2.0.2.0]

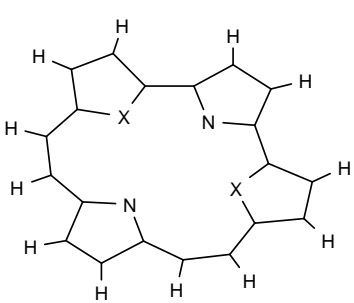

5a-X; [2-2.0.0]

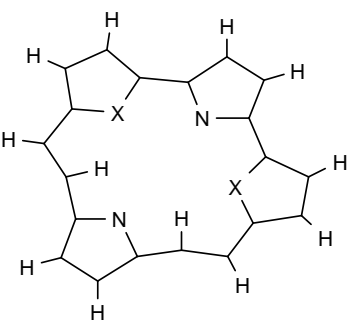

5e-X; $[2 \cdot 2 \cdot 0 \cdot 0]$

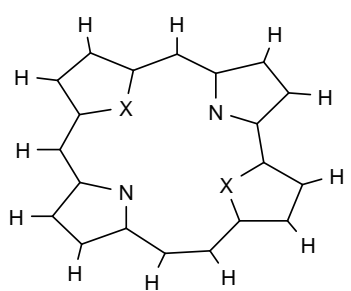

2a-X; [2.1.1.0]

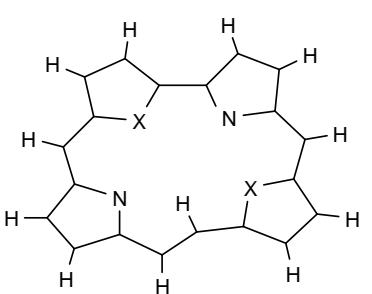

3b-X; [2.1.0.1]

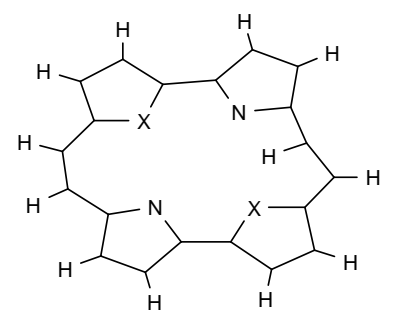

$4 c-X ;[2 \cdot 0 \cdot 2 \cdot 0]$

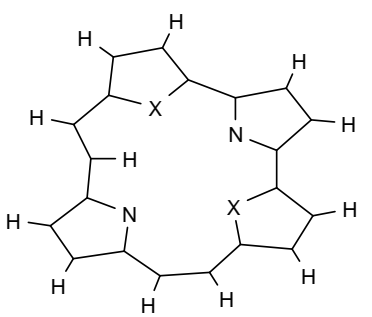

5b-X; [2.2.0.0]

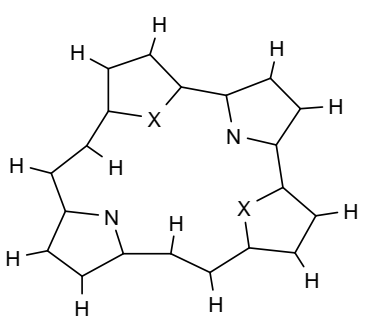

5f-X; $[2 \cdot 2 \cdot 0 \cdot 0]$

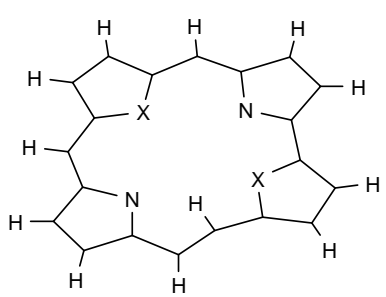

2b-X; [2·1·1·0]

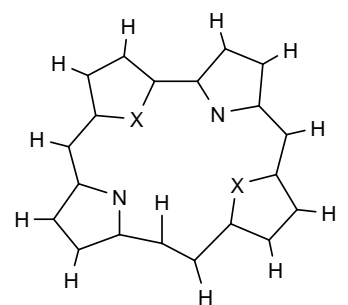

$3 c-X ;[2 \cdot 1 \cdot 0 \cdot 1]$

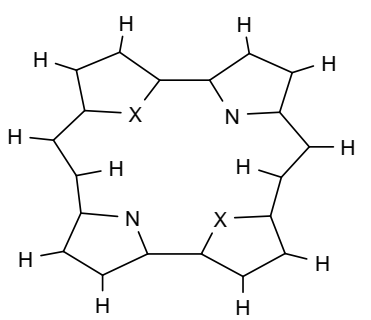

4d-X; [2.0.2.0]

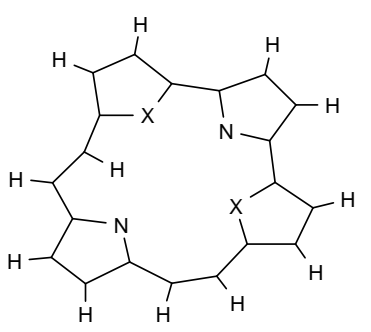

5c-X; [2.2.0.0]

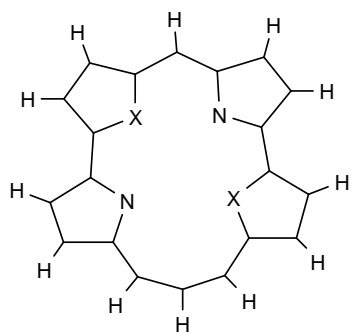

6a-X; $[3 \cdot 0 \cdot 1 \cdot 0]$

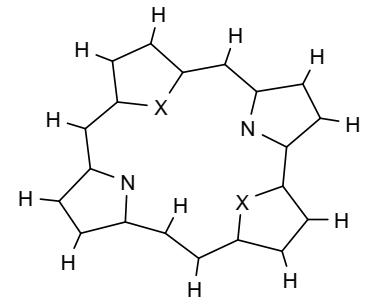

2c-X; [2.1·1·0]

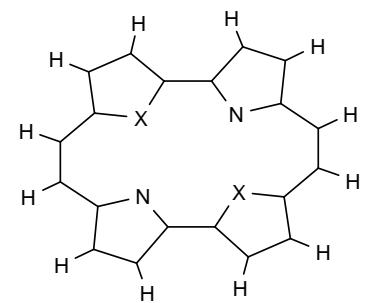

4a-X; [2.0.2.0]

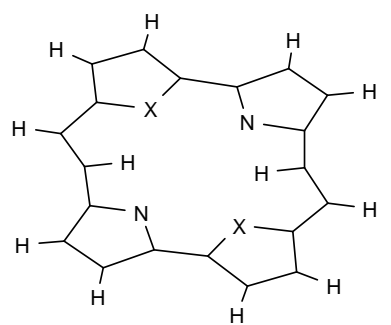

4e-X; $[2 \cdot 0 \cdot 2 \cdot 0]$

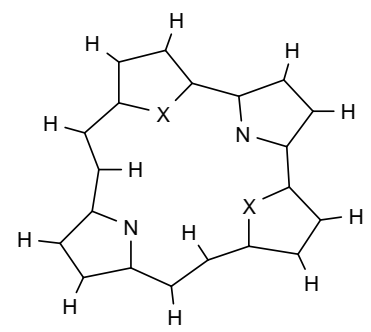

5d-X; [2·2.0.0]

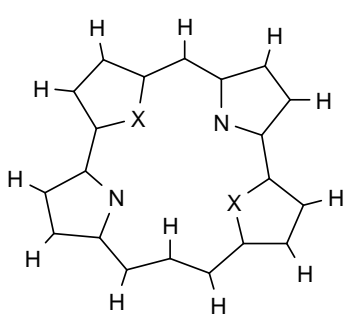

6b-X; [3.0.1.0]

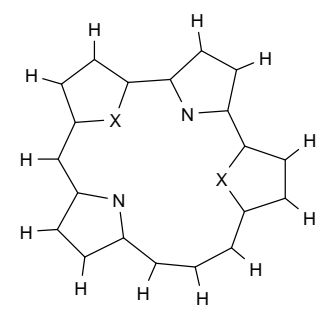

7a-X; [3.1.0.0]

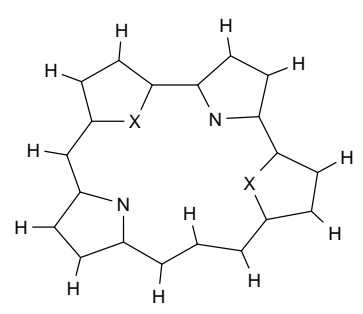

7b-X; [3.1.0.0]

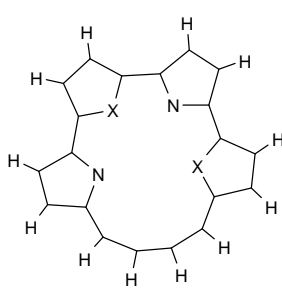

8a-X; [4.0.0.0]

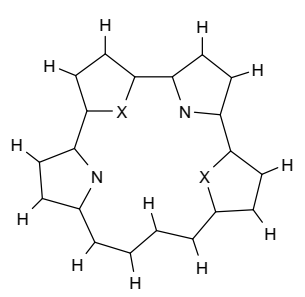

8b-X; [4-0.0.0]

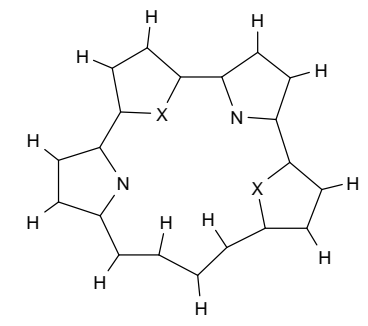

8c-X; $[4 \cdot 0 \cdot 0 \cdot 0]$

Scheme 1. Anti porphyrin $\left(\mathrm{C}_{20} \mathrm{X}_{2} \mathrm{~N}_{2} \mathrm{H}_{12}\right)$ isomers; here $\mathrm{X}=\mathrm{NH}, \mathrm{O}$, S. The projections are the AM1 optimised geometry of the parent $\left(\mathrm{C}_{20} \mathrm{~N}_{4} \mathrm{H}_{14}\right)$ isomers. 


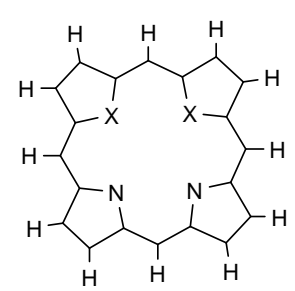

$\mathbf{1}^{\prime}-\mathrm{X} ;[1 \cdot 1 \cdot 1 \cdot 1]$

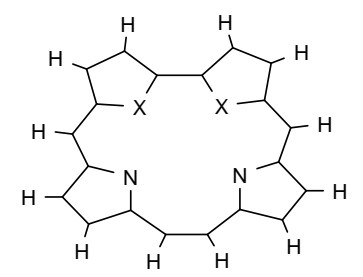

$3 \mathbf{a}^{\prime}-\mathrm{X} ;[2 \cdot 1 \cdot 0 \cdot 1]$

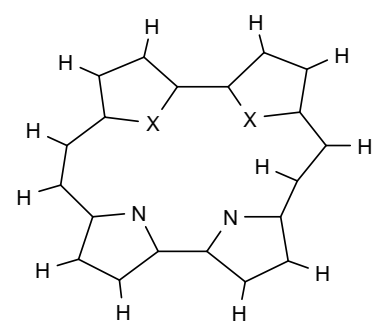

$4 b^{\prime}-X ;[2 \cdot 0 \cdot 2 \cdot 0]$

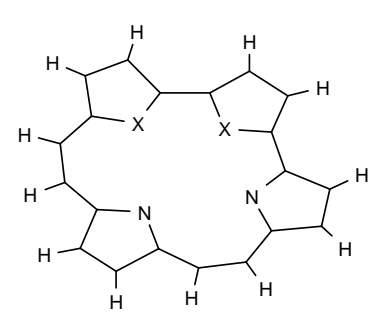

$5 \mathbf{a}^{\prime}-\mathrm{X} ;[2 \cdot 2 \cdot 0 \cdot 0]$

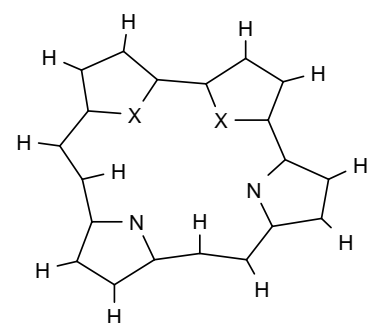

$5 \mathbf{e}^{\prime}-\mathrm{X} ;[2 \cdot 2 \cdot 0 \cdot 0]$

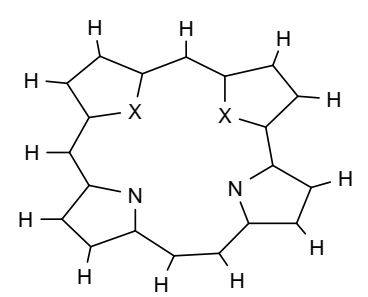

$2 \mathbf{a}^{\prime}-X ;[2 \cdot 1 \cdot 1 \cdot 0]$

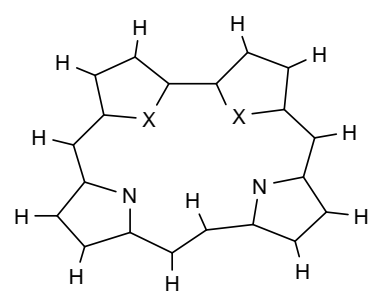

$3 \mathbf{b}^{\prime}-\mathrm{X} ;[2 \cdot 1 \cdot 0 \cdot 1]$

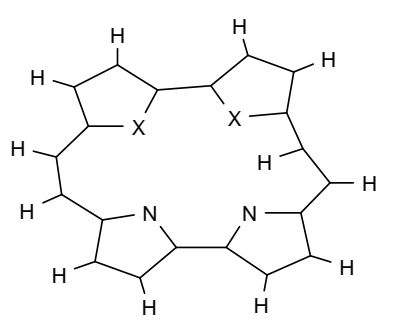

$4 \mathbf{c}^{\prime}-X ;[2 \cdot 0 \cdot 2 \cdot 0]$

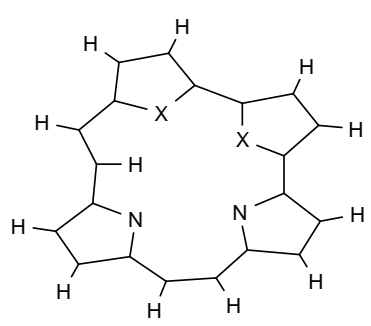

$5 \mathbf{b}^{\prime}-\mathrm{X}$; [2.2.0.0]

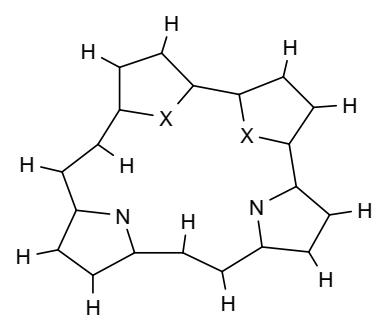

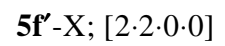

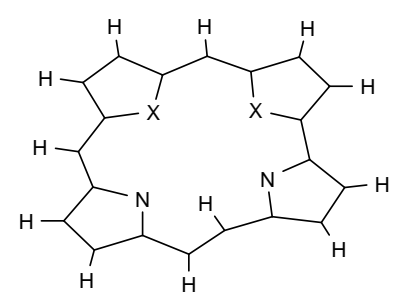

2b'-X; [2.1.1.0]

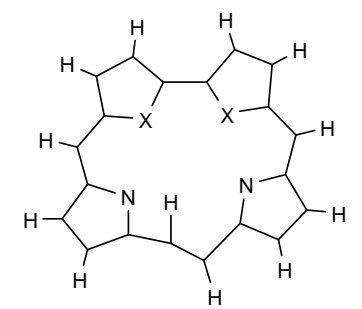

$3 \mathbf{c}^{\prime}-\mathrm{X} ;[2 \cdot 1 \cdot 0 \cdot 1]$

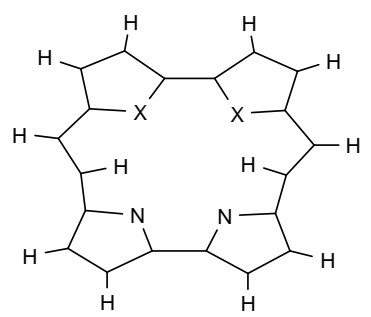

$4 d^{\prime}-X ;[2 \cdot 0 \cdot 2 \cdot 0]$

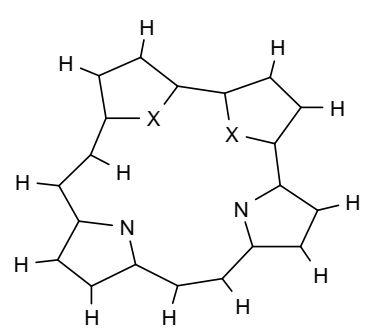

$5 \mathbf{c}^{\prime}-\mathrm{X}$; [2.2.0.0]

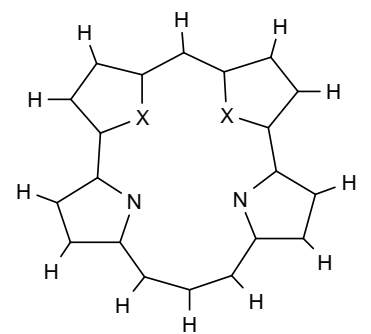

$\mathbf{6 a}^{\prime}-\mathrm{X} ;[3 \cdot 0 \cdot 1 \cdot 0]$

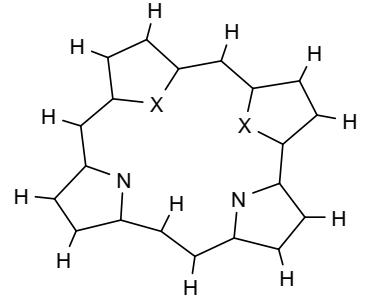

$2 \mathbf{c}^{\prime}-\mathrm{X}$; [2.1.1.0]

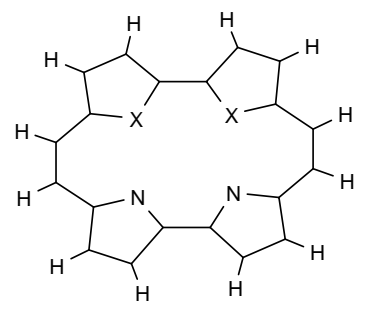

$4 \mathbf{a}^{\prime}-\mathrm{X} ;[2 \cdot 0 \cdot 2 \cdot 0]$

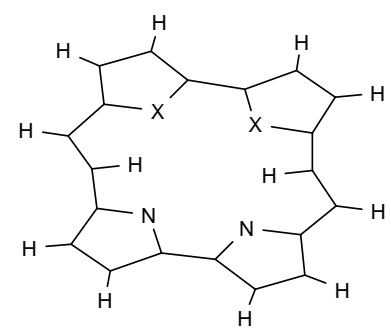

$4 \mathrm{e}^{\prime}-\mathrm{X} ;[2 \cdot 0 \cdot 2 \cdot 0]$

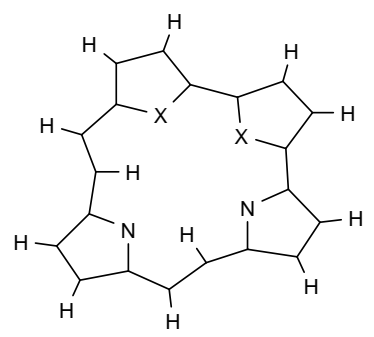

$5 d^{\prime}-X ;[2 \cdot 2 \cdot 0 \cdot 0]$

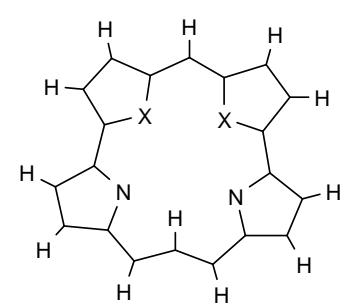

6b' $-\mathrm{X}$; [3.0.1.0]

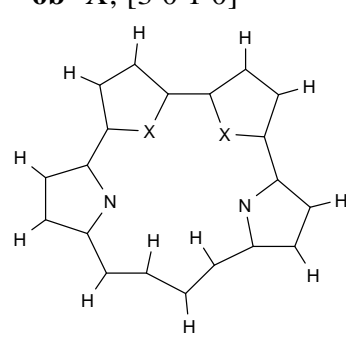

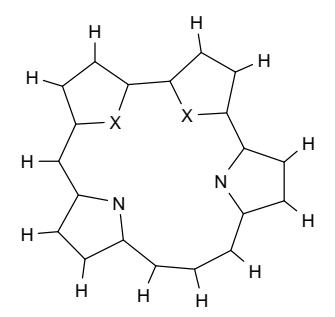

$7 \mathbf{a}^{\prime}-\mathrm{X} ;[3 \cdot 1 \cdot 0 \cdot 0]$

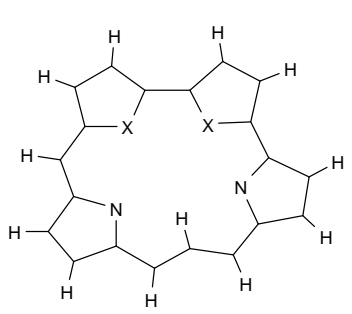

$7 \mathbf{b}^{\prime}-\mathrm{X} ;[3 \cdot 1 \cdot 0 \cdot 0]$

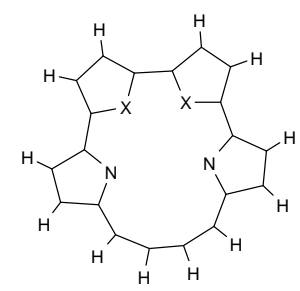

$8 \mathbf{a}^{\prime}-\mathrm{X} ;[4 \cdot 0 \cdot 0 \cdot 0]$

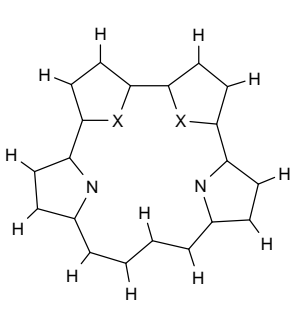

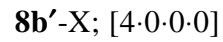

$8 \mathbf{c}^{\prime}-\mathrm{X}$; [4-0.0.0]

Scheme 2. Syn porphyrin $\left(\mathrm{C}_{20} \mathrm{X}_{2} \mathrm{~N}_{2} \mathrm{H}_{12}\right)$ isomers; here $\mathrm{X}=\mathrm{NH}, \mathrm{O}, \mathrm{S}$. The projections are the AM1 optimised geometry of the parent $\left(\mathrm{C}_{20} \mathrm{~N}_{4} \mathrm{H}_{14}\right)$ isomers. 
Table 1. Total energies, relative energies and distortion energies of anti tautomers of porphyrin isomers obtained at semiempirical (AM1, PM3) and DFT (B3LYP/6-31G*//AM1) levels of theory. Total energies are in hartrees, and relative energies and distortion energies (DE) are in $\mathrm{kcal} \mathrm{mol}^{-1}$.

\begin{tabular}{|c|c|c|c|c|c|c|c|c|c|}
\hline \multirow[b]{2}{*}{ Structure } & \multicolumn{3}{|c|}{ AM1 } & \multicolumn{3}{|c|}{ PM3 } & \multicolumn{3}{|c|}{ B3LYP/6-31G*//AM1 } \\
\hline & $\mathrm{HF}$ & $\Delta E$ & $\mathrm{DE}$ & $\mathrm{HF}$ & $\Delta E$ & DE & $\mathrm{HF}$ & $\Delta E$ & $\mathrm{DE}$ \\
\hline 1-NH & $0 \cdot 38603$ & $0 \cdot 0$ & $7 \cdot 2$ & $0 \cdot 29627$ & $0 \cdot 0$ & $7 \cdot 8$ & $-989 \cdot 53061$ & $0 \cdot 0$ & $-5 \cdot 7$ \\
\hline $\mathbf{2 a}-\mathrm{NH}$ & $0 \cdot 39674$ & $6 \cdot 7$ & $0 \cdot 1$ & $0 \cdot 28893$ & $-4 \cdot 6$ & $0 \cdot 0$ & $-989 \cdot 52293$ & $4 \cdot 8$ & $23 \cdot 5$ \\
\hline 2b-NH & $0 \cdot 44034$ & $34 \cdot 1$ & $45 \cdot 3$ & $0 \cdot 33342$ & $23 \cdot 3$ & $25 \cdot 6$ & $-989 \cdot 45347$ & $48 \cdot 4$ & $7 \cdot 5$ \\
\hline $2 \mathrm{c}-\mathrm{NH}$ & $0 \cdot 44295$ & $35 \cdot 7$ & $53 \cdot 5$ & $0 \cdot 34817$ & $32 \cdot 6$ & $35 \cdot 7$ & $-989 \cdot 44358$ & $54 \cdot 6$ & $4 \cdot 1$ \\
\hline 3a-NH & $0 \cdot 40767$ & $13 \cdot 6$ & $1 \cdot 3$ & $0 \cdot 30264$ & $4 \cdot 0$ & $0 \cdot 0$ & $-989 \cdot 50370$ & $16 \cdot 9$ & $-6 \cdot 6$ \\
\hline 3b-NH & $0 \cdot 43412$ & $30 \cdot 2$ & $20 \cdot 1$ & $0 \cdot 31657$ & $12 \cdot 7$ & $12 \cdot 3$ & $-989 \cdot 46921$ & $38 \cdot 5$ & $-7 \cdot 3$ \\
\hline 4a-NH & $0 \cdot 40785$ & $13 \cdot 7$ & $0 \cdot 2$ & $0 \cdot 29907$ & $1 \cdot 8$ & $5 \cdot 5$ & $-989 \cdot 52051$ & $6 \cdot 3$ & $-11 \cdot 3$ \\
\hline 4b-NH & 0.44252 & $35 \cdot 4$ & $67 \cdot 1$ & $0 \cdot 34120$ & $28 \cdot 2$ & $44 \cdot 9$ & -989.42659 & $65 \cdot 3$ & $9 \cdot 4$ \\
\hline 4c-NH & $0 \cdot 44167$ & 34.9 & $73 \cdot 3$ & $0 \cdot 34050$ & $27 \cdot 8$ & $52 \cdot 7$ & $-989 \cdot 42973$ & $63 \cdot 3$ & $15 \cdot 0$ \\
\hline 4d-NH & 0.49808 & $70 \cdot 3$ & $96 \cdot 5$ & $0 \cdot 38937$ & $58 \cdot 4$ & $59 \cdot 0$ & $-989 \cdot 37515$ & $97 \cdot 6$ & $19 \cdot 4$ \\
\hline 5a-NH & $0 \cdot 45192$ & $41 \cdot 4$ & $13 \cdot 2$ & $0 \cdot 35148$ & $34 \cdot 6$ & $5 \cdot 2$ & $-989 \cdot 45527$ & $47 \cdot 3$ & $-11 \cdot 1$ \\
\hline 5b-NH & 0.47880 & $58 \cdot 2$ & $41 \cdot 4$ & $0 \cdot 37176$ & $47 \cdot 3$ & $20 \cdot 6$ & $-989 \cdot 40801$ & $76 \cdot 9$ & $-15 \cdot 7$ \\
\hline 5c-NH & 0.45986 & $46 \cdot 3$ & $74 \cdot 9$ & $0 \cdot 35558$ & $37 \cdot 2$ & $44 \cdot 9$ & $-989 \cdot 41503$ & $72 \cdot 5$ & $10 \cdot 4$ \\
\hline 5d-NH & $0 \cdot 50162$ & $72 \cdot 5$ & $90 \cdot 9$ & $0 \cdot 39106$ & $59 \cdot 5$ & $54 \cdot 1$ & $-989 \cdot 37615$ & $96 \cdot 9$ & $15 \cdot 3$ \\
\hline 5e-NH & $0 \cdot 50525$ & $74 \cdot 8$ & $96 \cdot 2$ & $0 \cdot 39618$ & $62 \cdot 7$ & $62 \cdot 7$ & $-989 \cdot 38513$ & $91 \cdot 3$ & $29 \cdot 2$ \\
\hline 6a-NH & 0.42558 & $24 \cdot 8$ & $6 \cdot 7$ & $0 \cdot 31572$ & $12 \cdot 2$ & $2 \cdot 4$ & $-989 \cdot 46654$ & $40 \cdot 2$ & $-20 \cdot 4$ \\
\hline 6b-NH & 0.43001 & $27 \cdot 6$ & $16 \cdot 4$ & $0 \cdot 31609$ & $12 \cdot 4$ & $7 \cdot 5$ & $-989 \cdot 47001$ & $38 \cdot 0$ & $-11 \cdot 0$ \\
\hline 7a-NH & $0 \cdot 45046$ & $40 \cdot 4$ & $22 \cdot 6$ & $0 \cdot 34421$ & $30 \cdot 1$ & $16 \cdot 8$ & $-989 \cdot 42980$ & $63 \cdot 3$ & $-19 \cdot 1$ \\
\hline 7b-NH & $0 \cdot 44744$ & $38 \cdot 5$ & $24 \cdot 5$ & $0 \cdot 33716$ & $25 \cdot 7$ & $11 \cdot 6$ & $-989 \cdot 44848$ & $51 \cdot 5$ & $-16 \cdot 3$ \\
\hline 8a-NH & $0 \cdot 48305$ & $60 \cdot 9$ & $52 \cdot 4$ & $0 \cdot 36428$ & $42 \cdot 7$ & $40 \cdot 6$ & $-989 \cdot 37871$ & $95 \cdot 3$ & $-14 \cdot 0$ \\
\hline 8b-NH & 0.47378 & $55 \cdot 1$ & $23 \cdot 1$ & $0 \cdot 36332$ & $42 \cdot 1$ & $4 \cdot 1$ & -989.40999 & $75 \cdot 7$ & $-29 \cdot 9$ \\
\hline 8c-NH & $0 \cdot 50567$ & $75 \cdot 1$ & $84 \cdot 8$ & $0 \cdot 39644$ & $62 \cdot 9$ & $49 \cdot 8$ & $-989 \cdot 38282$ & $92 \cdot 7$ & $16 \cdot 9$ \\
\hline
\end{tabular}

Table 2. Total energies, relative energies and distortion energies of anti tautomers of core-modified oxaporphyrin isomers obtained at semiempirical (AM1, PM3) and DFT (B3LYP/6-31G*//AM1) levels of theory. Total energies are in hartrees and relative energies and distortion energies (DE) are in kcal mol ${ }^{-1}$.

\begin{tabular}{|c|c|c|c|c|c|c|c|c|c|}
\hline \multirow[b]{2}{*}{ Structure } & \multicolumn{3}{|c|}{ AM1 } & \multicolumn{3}{|c|}{ PM3 } & \multicolumn{3}{|c|}{ B3LYP/6-31G*//AM1 } \\
\hline & $\mathrm{HF}$ & $\Delta E$ & $\mathrm{DE}$ & $\mathrm{HF}$ & $\Delta E$ & $\mathrm{DE}$ & $\mathrm{HF}$ & $\Delta E$ & $\mathrm{DE}$ \\
\hline $1-\mathrm{O}$ & $0 \cdot 31190$ & $0 \cdot 0$ & $11 \cdot 8$ & $0 \cdot 23261$ & $0 \cdot 0$ & $13 \cdot 6$ & $-1029 \cdot 18965$ & $0 \cdot 0$ & $-6 \cdot 5$ \\
\hline $\mathbf{2 a}-\mathrm{O}$ & $0 \cdot 32072$ & $5 \cdot 5$ & $0 \cdot 1$ & $0 \cdot 23703$ & $2 \cdot 8$ & $0 \cdot 0$ & $-1029 \cdot 17982$ & $6 \cdot 2$ & $-2 \cdot 0$ \\
\hline $\mathbf{2 b}-\mathrm{O}$ & $0 \cdot 36026$ & $30 \cdot 3$ & $26 \cdot 6$ & $0 \cdot 26883$ & $22 \cdot 7$ & $17 \cdot 2$ & $-1029 \cdot 12169$ & $42 \cdot 6$ & $-2 \cdot 2$ \\
\hline $2 c-O$ & $0 \cdot 34385$ & $20 \cdot 0$ & $39 \cdot 6$ & $0 \cdot 25947$ & $16 \cdot 9$ & $29 \cdot 0$ & $-1029 \cdot 14049$ & $30 \cdot 9$ & $12 \cdot 3$ \\
\hline $3 \mathbf{a}-\mathrm{O}$ & $0 \cdot 33046$ & $11 \cdot 6$ & $1 \cdot 2$ & $0 \cdot 24425$ & $7 \cdot 3$ & $1 \cdot 6$ & $-1029 \cdot 14671$ & $27 \cdot 0$ & $-17 \cdot 4$ \\
\hline 3b-O & $0 \cdot 34212$ & $19 \cdot 0$ & $10 \cdot 3$ & $0 \cdot 25472$ & $13 \cdot 9$ & $5 \cdot 9$ & $-1029 \cdot 14620$ & $27 \cdot 3$ & $-6 \cdot 9$ \\
\hline $4 \mathbf{a}-\mathrm{O}$ & $0 \cdot 32623$ & $9 \cdot 0$ & $11 \cdot 8$ & $0 \cdot 24401$ & $7 \cdot 2$ & $12 \cdot 4$ & $-1029 \cdot 16788$ & $13 \cdot 7$ & $-12 \cdot 7$ \\
\hline $4 b-O$ & $0 \cdot 35604$ & $27 \cdot 7$ & $49 \cdot 3$ & $0 \cdot 26840$ & $22 \cdot 5$ & $34 \cdot 5$ & $-1029 \cdot 11012$ & $49 \cdot 9$ & $9 \cdot 6$ \\
\hline $4 c-O$ & $0 \cdot 34624$ & $21 \cdot 5$ & $58 \cdot 7$ & $0 \cdot 26240$ & $18 \cdot 7$ & $41 \cdot 4$ & $-1029 \cdot 13714$ & $33 \cdot 0$ & $27 \cdot 9$ \\
\hline $4 d-O$ & $0 \cdot 39801$ & $54 \cdot 0$ & $64 \cdot 1$ & $0 \cdot 30650$ & $46 \cdot 4$ & $40 \cdot 3$ & $-1029 \cdot 07557$ & $71 \cdot 6$ & $15 \cdot 6$ \\
\hline $5 \mathbf{a}-\mathrm{O}$ & $0 \cdot 36801$ & $35 \cdot 2$ & $9 \cdot 0$ & $0 \cdot 27796$ & $28 \cdot 5$ & $6 \cdot 5$ & $-1029 \cdot 11266$ & $48 \cdot 5$ & $-16 \cdot 8$ \\
\hline $5 b-O$ & $0 \cdot 35604$ & $27 \cdot 7$ & $35 \cdot 2$ & $0 \cdot 26840$ & $22 \cdot 5$ & $24 \cdot 1$ & $-1029 \cdot 11012$ & $49 \cdot 9$ & $-6 \cdot 4$ \\
\hline $5 c-O$ & $0 \cdot 36853$ & $35 \cdot 5$ & $50 \cdot 9$ & $0 \cdot 28139$ & $30 \cdot 6$ & $32 \cdot 0$ & $-1029 \cdot 10129$ & $55 \cdot 4$ & $10 \cdot 6$ \\
\hline $5 d-O$ & $0 \cdot 40191$ & $56 \cdot 4$ & $58 \cdot 9$ & $0 \cdot 30959$ & $48 \cdot 3$ & $37 \cdot 3$ & $-1029 \cdot 07537$ & $71 \cdot 7$ & $12 \cdot 7$ \\
\hline $5 e-O$ & $0 \cdot 39478$ & $52 \cdot 0$ & $73 \cdot 4$ & $0 \cdot 30270$ & $44 \cdot 0$ & $50 \cdot 3$ & $-1029 \cdot 08580$ & $65 \cdot 2$ & $26 \cdot 6$ \\
\hline $\mathbf{6 a - O}$ & $0 \cdot 33908$ & $17 \cdot 1$ & $7 \cdot 4$ & $0 \cdot 25107$ & $11 \cdot 6$ & $5 \cdot 1$ & $-1029 \cdot 13031$ & $37 \cdot 2$ & $-17 \cdot 7$ \\
\hline 6b-O & $0 \cdot 33868$ & $16 \cdot 8$ & $6 \cdot 7$ & $0 \cdot 24942$ & $10 \cdot 5$ & $3 \cdot 3$ & $-1029 \cdot 15070$ & $24 \cdot 4$ & $-12 \cdot 4$ \\
\hline $7 \mathbf{a}-\mathrm{O}$ & $0 \cdot 36132$ & $31 \cdot 0$ & $17 \cdot 6$ & $0 \cdot 27307$ & $25 \cdot 4$ & $13 \cdot 9$ & $-1029 \cdot 10600$ & $52 \cdot 5$ & $-13 \cdot 0$ \\
\hline $7 \mathbf{b}-\mathrm{O}$ & $0 \cdot 35130$ & $24 \cdot 7$ & $9 \cdot 7$ & $0 \cdot 26393$ & $19 \cdot 6$ & $4 \cdot 2$ & $-1029 \cdot 14291$ & $29 \cdot 3$ & $-8 \cdot 7$ \\
\hline $\mathbf{8 a}-\mathrm{O}$ & $0 \cdot 38060$ & $43 \cdot 1$ & $46 \cdot 9$ & $0 \cdot 28752$ & $34 \cdot 5$ & $35 \cdot 3$ & $-1029 \cdot 08089$ & $68 \cdot 3$ & $3 \cdot 9$ \\
\hline 8b-O & $0 \cdot 36459$ & $33 \cdot 1$ & $13 \cdot 7$ & $0 \cdot 27359$ & $25 \cdot 7$ & $4 \cdot 6$ & $-1029 \cdot 12715$ & $39 \cdot 2$ & $-11 \cdot 9$ \\
\hline $8 c-O$ & $0 \cdot 41187$ & $62 \cdot 7$ & $56 \cdot 9$ & $0 \cdot 31984$ & $54 \cdot 7$ & $32 \cdot 9$ & $-1029 \cdot 06860$ & $76 \cdot 0$ & $13 \cdot 0$ \\
\hline
\end{tabular}


Table 3. Total energies, relative energies and distortion energies of anti tautomers of core-modified thiaporphyrin isomers obtained at semiempirical (AM1, PM3) and DFT (B3LYP/6-31G*//AM1) levels of theory. Total energies are in hartrees and relative energies and distortion energies (DE) are in kcal $\mathrm{mol}^{-1}$.

\begin{tabular}{|c|c|c|c|c|c|c|c|c|c|}
\hline \multirow[b]{2}{*}{ Structure } & \multicolumn{3}{|c|}{ AM1 } & \multicolumn{3}{|c|}{ PM3 } & \multicolumn{3}{|c|}{ B3LYP/6-31G*//AM1 } \\
\hline & $\mathrm{HF}$ & $\Delta E$ & $\mathrm{DE}$ & $\mathrm{HF}$ & $\Delta E$ & $\mathrm{DE}$ & $\mathrm{HF}$ & $\Delta E$ & $\mathrm{DE}$ \\
\hline 1-S & $0 \cdot 41422$ & $0 \cdot 0$ & $10 \cdot 3$ & $0 \cdot 36641$ & $0 \cdot 0$ & $10 \cdot 4$ & $-1675 \cdot 14507$ & $0 \cdot 0$ & $-6 \cdot 4$ \\
\hline $\mathbf{2 a}-\mathrm{S}$ & $0 \cdot 42709$ & $8 \cdot 1$ & $4 \cdot 6$ & $0 \cdot 36904$ & $1 \cdot 6$ & $0 \cdot 0$ & $-1675 \cdot 10476$ & $25 \cdot 3$ & $-10 \cdot 0$ \\
\hline $2 \mathbf{b}-\mathrm{S}$ & 0.46559 & $32 \cdot 2$ & $46 \cdot 0$ & $0 \cdot 40832$ & $26 \cdot 3$ & $33 \cdot 2$ & $-1675 \cdot 05825$ & $54 \cdot 5$ & $14 \cdot 5$ \\
\hline $\mathbf{3 a}-\mathrm{S}$ & $0 \cdot 43415$ & $12 \cdot 5$ & $6 \cdot 8$ & $0 \cdot 37784$ & $7 \cdot 2$ & $9 \cdot 5$ & $-1675 \cdot 10541$ & $24 \cdot 9$ & $-5 \cdot 6$ \\
\hline $\mathbf{3 b}-\mathrm{S}$ & $0 \cdot 45676$ & $26 \cdot 7$ & $31 \cdot 0$ & $0 \cdot 39828$ & $20 \cdot 0$ & $14 \cdot 7$ & $-1675 \cdot 05890$ & $54 \cdot 1$ & $0 \cdot 4$ \\
\hline $4 \mathbf{a}-\mathrm{S}$ & $0 \cdot 43134$ & $10 \cdot 7$ & $21 \cdot 2$ & $0 \cdot 35245$ & $-8 \cdot 8$ & $6 \cdot 1$ & $-1675 \cdot 09129$ & $33 \cdot 8$ & $-16 \cdot 3$ \\
\hline $4 b-S$ & $0 \cdot 45262$ & $24 \cdot 1$ & $78 \cdot 2$ & $0 \cdot 39172$ & $15 \cdot 9$ & $68 \cdot 4$ & $-1675 \cdot 05468$ & $56 \cdot 7$ & $35 \cdot 1$ \\
\hline $4 c-S$ & $0 \cdot 46528$ & $32 \cdot 0$ & $75 \cdot 5$ & $0 \cdot 41344$ & $29 \cdot 5$ & $58 \cdot 5$ & $-1675 \cdot 05116$ & 58.9 & $35 \cdot 3$ \\
\hline 4d-S & $0 \cdot 50840$ & $59 \cdot 1$ & $105 \cdot 8$ & 0.44739 & $50 \cdot 8$ & $74 \cdot 7$ & $-1675 \cdot 00310$ & $89 \cdot 1$ & $51 \cdot 4$ \\
\hline $4 e-S$ & 0.52116 & $67 \cdot 1$ & $100 \cdot 0$ & 0.45961 & $58 \cdot 5$ & $64 \cdot 1$ & -1674.99658 & $93 \cdot 2$ & $49 \cdot 4$ \\
\hline $5 \mathbf{a}-\mathrm{S}$ & $0 \cdot 46274$ & $30 \cdot 4$ & $19 \cdot 8$ & $0 \cdot 40689$ & $25 \cdot 4$ & $14 \cdot 6$ & $-1675 \cdot 07332$ & $45 \cdot 0$ & $-5 \cdot 5$ \\
\hline $5 \mathbf{b}-\mathrm{S}$ & 0.49458 & $50 \cdot 4$ & $51 \cdot 8$ & 0.43359 & $42 \cdot 2$ & $33 \cdot 2$ & $-1675 \cdot 01080$ & $84 \cdot 3$ & $5 \cdot 6$ \\
\hline $5 c-S$ & 0.46943 & $34 \cdot 7$ & $88 \cdot 4$ & $0 \cdot 41127$ & $28 \cdot 1$ & $59 \cdot 8$ & $-1675 \cdot 03866$ & $66 \cdot 8$ & $41 \cdot 1$ \\
\hline $5 d-S$ & $0 \cdot 51088$ & $60 \cdot 7$ & $103 \cdot 2$ & $0 \cdot 44285$ & $48 \cdot 0$ & $77 \cdot 8$ & -1674.99322 & $95 \cdot 3$ & $44 \cdot 7$ \\
\hline $5 e-S$ & $0 \cdot 52066$ & $66 \cdot 8$ & $101 \cdot 7$ & 0.45988 & $58 \cdot 7$ & $73 \cdot 6$ & $-1675 \cdot 00793$ & $86 \cdot 1$ & $57 \cdot 0$ \\
\hline 6a-S & 0.44878 & $21 \cdot 7$ & $17 \cdot 8$ & $0 \cdot 39548$ & $18 \cdot 2$ & $2 \cdot 0$ & $-1675 \cdot 07694$ & $42 \cdot 8$ & $-2 \cdot 1$ \\
\hline 6b-S & $0 \cdot 45709$ & $26 \cdot 9$ & $22 \cdot 1$ & $0 \cdot 39627$ & $18 \cdot 7$ & $14 \cdot 6$ & $-1675 \cdot 05514$ & $56 \cdot 4$ & $-13 \cdot 9$ \\
\hline $7 \mathbf{a}-S$ & $0 \cdot 47029$ & $35 \cdot 2$ & $37 \cdot 6$ & $0 \cdot 41502$ & $30 \cdot 5$ & $31 \cdot 1$ & $-1675 \cdot 03943$ & $66 \cdot 3$ & $-1 \cdot 1$ \\
\hline $7 b-S$ & $0 \cdot 46773$ & $33 \cdot 6$ & $35 \cdot 6$ & $0 \cdot 41110$ & $28 \cdot 0$ & $23 \cdot 4$ & $-1675 \cdot 05560$ & $56 \cdot 1$ & $4 \cdot 5$ \\
\hline $8 \mathbf{a}-\mathrm{S}$ & $0 \cdot 49127$ & $48 \cdot 3$ & $72 \cdot 0$ & $0 \cdot 42688$ & $37 \cdot 9$ & $56 \cdot 2$ & $-1675 \cdot 00766$ & $86 \cdot 2$ & $18 \cdot 3$ \\
\hline 8b-S & $0 \cdot 48108$ & $42 \cdot 0$ & $44 \cdot 2$ & $0 \cdot 42186$ & $34 \cdot 8$ & $29 \cdot 9$ & $-1675 \cdot 04447$ & $63 \cdot 1$ & $6 \cdot 5$ \\
\hline $8 c-S$ & 0.52320 & $68 \cdot 4$ & $96 \cdot 9$ & 0.46004 & $58 \cdot 8$ & $68 \cdot 9$ & -1674.98701 & $99 \cdot 2$ & $37 \cdot 5$ \\
\hline
\end{tabular}

the oxa- and thia-disubstituted analogues respectively. Unexpectedly, semiempirical AM1 and PM3 methods designate all isomers including the parent porphyrin as non-planar and they are characterized as local minima, highlighting the inadequacy of semiempirical methodology in treating porphyrintype molecules. Clearly, at higher levels of theory, the parent molecule has planar minima. Thus, we intend to check the energy differences between the planar and minimum energy structures (distortion energy) at $\mathrm{B} 3 \mathrm{LYP} / 6-31 \mathrm{G}^{*}$ level also in addition to semiempirical levels to assess the performance of the semiempirical methods. Distortion energy may be taken as a measure of strain energy in the planar form due to steric congestion due to the core hydrogens and the angular strain. As out-of-plane puckering has been observed in expanded porphyrins, we also intend to gauge the performance of semiempirical methodologies in modelling the out-of-plane distortions in this class of compounds. While B3LYP/6-31G* single point calculations on AM1 stationary points show that quite a few structures have positive distortion energies, indicating that the puckered structures are more stable, some of them have negative distortion energies. The negative distortion energy at B3LYP level indicates that the semiempirical methods wrongly estimate that the isomers are puckered. Similar trends are also seen in oxa- and thia-core-modified analogues. Importantly, the magnitude of distortion energy also varies quite significantly at the three levels of theory considered. Thus, the present study clearly exposes the limitations of the semiempirical methodologies in modelling porphyrin-type molecules.

Significant variations in the relative energies and relative energy orderings are observed depending on the level of theory employed. Angle strain and steric repulsion between the inner protons appear to be the principal causative factors for the destabilization of the various isomeric forms. Obviously, direct pyrrolic linkages lead to higher angle strain and cause destabilization. Thus, expectedly, the isomers $\mathbf{8 a}-\mathrm{X}-\mathbf{8 c}-\mathrm{X}$ are among the least stable isomeric type in parent as well as the core-modified analogues. However, some other isomers, e.g., 4d-X, 5d-X and 5e-X, are also very unstable despite the smaller number of direct 
Table 4. Total energies, relative energies and distortion energies of syn tautomers of porphyrin isomers obtained at semiempirical (AM1, PM3) and DFT (B3LYP/6-31G*//AM1) levels of theory. Total energies are in hartrees and relative energies and distortion energies (DE) are in $\mathrm{kcal} \mathrm{mol}^{-1}$.

\begin{tabular}{|c|c|c|c|c|c|c|c|c|c|}
\hline \multirow[b]{2}{*}{ Structure } & \multicolumn{3}{|c|}{ AM1 } & \multicolumn{3}{|c|}{ PM3 } & \multicolumn{3}{|c|}{ B3LYP/6-31G*//AM1 } \\
\hline & $\mathrm{HF}$ & $\Delta E$ & $\mathrm{DE}$ & $\mathrm{HF}$ & $\Delta E$ & $\mathrm{DE}$ & $\mathrm{HF}$ & $\Delta E$ & $\mathrm{DE}$ \\
\hline $\mathbf{1}^{\prime}-\mathrm{NH}$ & $0 \cdot 39719$ & $0 \cdot 0$ & $7 \cdot 1$ & $0 \cdot 30747$ & $0 \cdot 0$ & $6 \cdot 7$ & $-989 \cdot 50293$ & $0 \cdot 0$ & $-11 \cdot 8$ \\
\hline $\mathbf{2} \mathbf{a}^{\prime}-\mathrm{NH}$ & $0 \cdot 41630$ & $12 \cdot 0$ & $10 \cdot 7$ & $0 \cdot 32642$ & $11 \cdot 9$ & 4.9 & $-989 \cdot 47144$ & $19 \cdot 8$ & $-8 \cdot 8$ \\
\hline $2 \mathbf{b}^{\prime}-\mathrm{NH}$ & 0.44377 & $29 \cdot 2$ & $55 \cdot 0$ & $0 \cdot 39750$ & $25 \cdot 1$ & $28 \cdot 7$ & $-989 \cdot 42245$ & $50 \cdot 5$ & $-1 \cdot 6$ \\
\hline $2 c^{\prime}-\mathrm{NH}$ & $0 \cdot 46334$ & $41 \cdot 5$ & $27 \cdot 7$ & $0 \cdot 35732$ & $31 \cdot 3$ & $11 \cdot 9$ & $-989 \cdot 42025$ & $51 \cdot 9$ & $-14 \cdot 3$ \\
\hline $3 \mathbf{a}^{\prime}-\mathrm{NH}$ & $0 \cdot 41025$ & $8 \cdot 2$ & $4 \cdot 0$ & $0 \cdot 31030$ & $1 \cdot 8$ & $0 \cdot 2$ & $-989 \cdot 48421$ & $11 \cdot 7$ & $-12 \cdot 2$ \\
\hline $3 \mathbf{b}^{\prime}-\mathrm{NH}$ & $0 \cdot 43853$ & $25 \cdot 9$ & $19 \cdot 8$ & $0 \cdot 32249$ & $9 \cdot 4$ & $11 \cdot 4$ & $-989 \cdot 44810$ & $34 \cdot 4$ & $-16 \cdot 9$ \\
\hline $3 \mathbf{c}^{\prime}-\mathrm{NH}$ & 0.43750 & $25 \cdot 3$ & $20 \cdot 5$ & $0 \cdot 32249$ & $9 \cdot 4$ & $11 \cdot 4$ & $-989 \cdot 45199$ & $32 \cdot 0$ & $-14 \cdot 4$ \\
\hline $4 \mathbf{a}^{\prime}-\mathrm{NH}$ & 0.40374 & $4 \cdot 1$ & $0 \cdot 5$ & 0.29456 & $-8 \cdot 1$ & $0 \cdot 0$ & $-989 \cdot 51055$ & $-4 \cdot 8$ & $-8 \cdot 6$ \\
\hline $4 \mathbf{b}^{\prime}-\mathrm{NH}$ & 0.46792 & $44 \cdot 4$ & $57 \cdot 9$ & $0 \cdot 36116$ & $33 \cdot 7$ & $40 \cdot 3$ & $-989 \cdot 41962$ & $52 \cdot 3$ & $18 \cdot 5$ \\
\hline $4 c^{\prime}-\mathrm{NH}$ & 0.46552 & $42 \cdot 9$ & $51 \cdot 6$ & $0 \cdot 36099$ & $33 \cdot 6$ & $34 \cdot 3$ & $-989 \cdot 41605$ & $54 \cdot 5$ & $6 \cdot 4$ \\
\hline $4 d^{\prime}-N H$ & 0.51160 & $71 \cdot 8$ & $96 \cdot 6$ & 0.40234 & $59 \cdot 5$ & $59 \cdot 6$ & $-989 \cdot 36018$ & 89.6 & $34 \cdot 5$ \\
\hline $4 e^{\prime}-N H$ & $0 \cdot 51886$ & $76 \cdot 3$ & $84 \cdot 4$ & 0.40537 & $61 \cdot 4$ & $54 \cdot 9$ & $-989 \cdot 35478$ & $93 \cdot 0$ & $19 \cdot 5$ \\
\hline $5 \mathbf{a}^{\prime}-\mathrm{NH}$ & $0 \cdot 43946$ & $26 \cdot 5$ & $20 \cdot 3$ & $0 \cdot 33845$ & $19 \cdot 4$ & $13 \cdot 2$ & $-989 \cdot 42962$ & $46 \cdot 0$ & $-21 \cdot 0$ \\
\hline $5 \mathbf{b}^{\prime}-\mathrm{NH}$ & 0.47351 & $47 \cdot 9$ & $55 \cdot 0$ & $0 \cdot 36809$ & $38 \cdot 0$ & $37 \cdot 7$ & $-989 \cdot 39527$ & $67 \cdot 6$ & $-1 \cdot 3$ \\
\hline $5 c^{\prime}-\mathrm{NH}$ & $0 \cdot 46921$ & $45 \cdot 2$ & $78 \cdot 8$ & $0 \cdot 36459$ & $35 \cdot 8$ & $54 \cdot 2$ & $-989 \cdot 40386$ & $62 \cdot 2$ & $16 \cdot 0$ \\
\hline $5 d^{\prime}-N H$ & 0.49811 & $63 \cdot 3$ & $90 \cdot 7$ & $0 \cdot 38604$ & $49 \cdot 3$ & $54 \cdot 6$ & $-989 \cdot 36116$ & 89.0 & $12 \cdot 2$ \\
\hline $5 e^{\prime}-\mathrm{NH}$ & $0 \cdot 49168$ & $59 \cdot 3$ & $95 \cdot 8$ & $0 \cdot 38194$ & $46 \cdot 7$ & $63 \cdot 2$ & $-989 \cdot 37384$ & $81 \cdot 0$ & $98 \cdot 2$ \\
\hline $5 f^{\prime}-\mathrm{NH}$ & 0.49746 & 62.9 & $91 \cdot 1$ & $0 \cdot 38835$ & $50 \cdot 7$ & $53 \cdot 1$ & $-989 \cdot 37113$ & $82 \cdot 7$ & $18 \cdot 4$ \\
\hline $\mathbf{6 a}^{\prime}-\mathrm{NH}$ & $0 \cdot 43148$ & $21 \cdot 5$ & $7 \cdot 0$ & $0 \cdot 32417$ & $10 \cdot 5$ & $1 \cdot 8$ & $-989 \cdot 44685$ & $35 \cdot 2$ & $-27 \cdot 6$ \\
\hline $\mathbf{6} \mathbf{b}^{\prime}-\mathrm{NH}$ & 0.43564 & $24 \cdot 1$ & $19 \cdot 3$ & $0 \cdot 32548$ & $11 \cdot 3$ & $39 \cdot 4$ & $-989 \cdot 45055$ & 32.9 & $-13 \cdot 8$ \\
\hline $7 \mathbf{a}^{\prime}-\mathrm{NH}$ & 0.44954 & $32 \cdot 8$ & $25 \cdot 7$ & $0 \cdot 34491$ & $23 \cdot 5$ & $17 \cdot 1$ & $-989 \cdot 41439$ & $55 \cdot 6$ & $-16 \cdot 6$ \\
\hline $7 \mathbf{b}^{\prime}-\mathrm{NH}$ & 0.44968 & $32 \cdot 9$ & $28 \cdot 8$ & $0 \cdot 34555$ & $23 \cdot 9$ & $14 \cdot 0$ & $-989 \cdot 43074$ & $45 \cdot 3$ & $-10 \cdot 3$ \\
\hline $8 \mathbf{a}^{\prime}-\mathrm{NH}$ & $0 \cdot 47213$ & $47 \cdot 0$ & $68 \cdot 5$ & $0 \cdot 36437$ & $35 \cdot 7$ & $48 \cdot 5$ & $-989 \cdot 38603$ & $73 \cdot 4$ & $-0 \cdot 5$ \\
\hline $\mathbf{8 b ^ { \prime } - N H}$ & 0.45405 & $35 \cdot 7$ & $37 \cdot 2$ & $0 \cdot 34603$ & $24 \cdot 2$ & $16 \cdot 3$ & $-989 \cdot 43330$ & $43 \cdot 7$ & $-13 \cdot 2$ \\
\hline $8 c^{\prime}-N H$ & $0 \cdot 49286$ & $60 \cdot 0$ & $88 \cdot 6$ & $0 \cdot 38213$ & $46 \cdot 8$ & $50 \cdot 9$ & $-989 \cdot 38187$ & $76 \cdot 0$ & $18 \cdot 2$ \\
\hline
\end{tabular}

pyrrolic linkages, indicating that the relative stability of the isomers is decided by several intricate factors.

Among the anti tautomeric structures of porphyrin isomers the parent isomer $\mathbf{1}$ was computed to be most stable at AM1 and B3LYP levels. Surprisingly, the PM3 method predicts that the isomer 2a-NH (hemiporphycene), which has $[2 \cdot 1 \cdot 1 \cdot 0]$ connectivity, and the double bond in the $Z$-form, is predicted to be the most stable. However, in the oxa- and thia-disubstituted analogues, the isomeric form $\mathbf{1}$ is computed to be most stable in all the three levels of theory. Attempts to locate distinct minimum energy structures for isomers $\mathbf{3 b}-\mathrm{NH}$ and $\mathbf{3 c}-\mathrm{NH}$ in corrphycene, 4d-NH and 4e- $\mathrm{NH}$ in porphycene, 5d-NH and 5f$\mathrm{NH}$ in $[2 \cdot 2 \cdot 0 \cdot 0]$ were futile, and all the putative structures collapse to other minima energy structures, as depicted in scheme 1. While the corresponding oxa-isomers follow exactly the same trend as the parent aza-isomers, among the thia-isomers, $2 \mathbf{b}-\mathbf{S}$ and $2 \mathbf{c}-\mathrm{S}$ and isomers $\mathbf{3 b}-\mathrm{S}$ and $\mathbf{3 c}-\mathrm{S}$ collapse to the same structure. However, in contrast to the parent and oxa-analogues, $4 \mathbf{d}-\mathrm{S}$ and $\mathbf{4 e - S}$ have distinct minima on the potential energy surface. Tables 1-3 indicate the quantitative differences among the three theoretical methods in relative stabilities and distortion energies.

Figure 1 depicts the variation of the relative energies in porphyrin, oxaporphyrin and thiaporphyrin isomers. The relative energies and the distortion energies of the anti tautomer of oxa- and thia-porphyrin isomers are given in tables 2 and 3. A comparison of tables 2 and 3 with table 1 indicates that the trends in the relative energy orderings of the oxa- and thia-analogues are not drastically altered when compared to the parent porphyrin isomers. Figure 3 depicts the three-dimensional structures for the more distorted isomers in parent, oxa- and thiaporphyrins for the syn and anti forms.

In oxaporphyrin, isomers [3.0.1.0] and [3.1.0.0], $E$ isomers $6 \mathbf{b}-\mathrm{O}$ and $\mathbf{7 b}-\mathrm{O}$ are more stable than the $Z$ form $6 \mathbf{a}-\mathrm{O}$ and $7 \mathbf{a}-\mathrm{O}$ and are about 13 and $23 \mathrm{kcal} /$ mol more stable than the $Z$ isomer respectively. In isomers $[4 \cdot 0 \cdot 0 \cdot 0]$ the relative energies of the isomers 
Table 5. Total energies, relative energies and distortion energies of syn tautomers of coremodified oxaporphyrin isomers obtained at semiempirical (AM1, PM3) and DFT (B3LYP/ 6-31G*//AM1) levels of theory. Total energies are in hartrees and relative energies and distortion energies (DE) are in $\mathrm{kcal} \mathrm{mol}^{-1}$.

\begin{tabular}{|c|c|c|c|c|c|c|c|}
\hline \multirow[b]{2}{*}{ Structure } & \multicolumn{3}{|c|}{ AM1 } & \multicolumn{2}{|c|}{ PM3 } & \multicolumn{2}{|c|}{ B3LYP/6-31G*//AM1 } \\
\hline & $\mathrm{HF}$ & $\Delta E$ & $\mathrm{DE}$ & $\mathrm{HF}$ & $\Delta E$ & $\mathrm{HF}$ & $\Delta E$ \\
\hline $\mathbf{1}^{\prime}-\mathrm{O}$ & $0 \cdot 31476$ & $0 \cdot 0$ & $10 \cdot 0$ & $0 \cdot 23438$ & $0 \cdot 0$ & $-1029 \cdot 18455$ & $0 \cdot 0$ \\
\hline $2 \mathbf{a}^{\prime}-\mathrm{O}$ & $0 \cdot 32344$ & $5 \cdot 4$ & $0 \cdot 1$ & $0 \cdot 24496$ & $6 \cdot 6$ & $-1029 \cdot 16976$ & $9 \cdot 3$ \\
\hline $2 \mathbf{b}^{\prime}-\mathrm{O}$ & $0 \cdot 34475$ & $18 \cdot 8$ & $58 \cdot 6$ & $0 \cdot 26037$ & $16 \cdot 3$ & $-1029 \cdot 12910$ & $34 \cdot 8$ \\
\hline $2 c^{\prime}-O$ & $0 \cdot 37861$ & $40 \cdot 1$ & $25 \cdot 7$ & $0 \cdot 28431$ & $31 \cdot 3$ & $-1029 \cdot 10425$ & $50 \cdot 4$ \\
\hline $3 \mathbf{a}^{\prime}-\mathrm{O}$ & $0 \cdot 31588$ & 0.7 & $0 \cdot 1$ & $0 \cdot 23811$ & $2 \cdot 3$ & $-1029 \cdot 17111$ & $8 \cdot 4$ \\
\hline $3 \mathbf{b}^{\prime}-\mathrm{O}$ & $0 \cdot 34918$ & $21 \cdot 6$ & $20 \cdot 3$ & $0 \cdot 25915$ & $15 \cdot 5$ & $-1029 \cdot 13659$ & $30 \cdot 1$ \\
\hline $4 \mathbf{a}^{\prime}-\mathrm{O}$ & $0 \cdot 32121$ & $4 \cdot 1$ & $1 \cdot 5$ & $0 \cdot 23842$ & $2 \cdot 5$ & $-1029 \cdot 16336$ & $13 \cdot 3$ \\
\hline $4 b^{\prime}-O$ & $0 \cdot 37202$ & $35 \cdot 9$ & $43 \cdot 8$ & $0 \cdot 28563$ & $32 \cdot 2$ & $-1029 \cdot 09795$ & $54 \cdot 3$ \\
\hline $4 \mathbf{c}^{\prime}-\mathrm{O}$ & $0 \cdot 37207$ & $36 \cdot 0$ & $27 \cdot 6$ & $0 \cdot 28340$ & $30 \cdot 8$ & $-1029 \cdot 10547$ & $49 \cdot 6$ \\
\hline $4 d^{\prime}-O$ & $0 \cdot 39958$ & $53 \cdot 2$ & $74 \cdot 3$ & $0 \cdot 30971$ & $47 \cdot 3$ & $-1029 \cdot 07183$ & $70 \cdot 7$ \\
\hline $4 e^{\prime}-O$ & $0 \cdot 39990$ & $53 \cdot 4$ & $48 \cdot 5$ & $0 \cdot 30971$ & $47 \cdot 3$ & $-1029 \cdot 07598$ & $68 \cdot 1$ \\
\hline $5 \mathbf{a}^{\prime}-\mathrm{O}$ & 0.33674 & $13 \cdot 8$ & $7 \cdot 8$ & $0 \cdot 25271$ & $11 \cdot 5$ & $-1029 \cdot 12745$ & $35 \cdot 8$ \\
\hline $5 \mathbf{b}^{\prime}-\mathrm{O}$ & $0 \cdot 35544$ & $25 \cdot 5$ & $14 \cdot 6$ & $0 \cdot 27311$ & $24 \cdot 3$ & $-1029 \cdot 12248$ & $38 \cdot 1$ \\
\hline $5 c^{\prime}-O$ & $0 \cdot 36084$ & $28 \cdot 9$ & $23 \cdot 6$ & $0 \cdot 28005$ & $28 \cdot 7$ & $-1029 \cdot 10378$ & $50 \cdot 7$ \\
\hline $5 d^{\prime}-O$ & $0 \cdot 37858$ & $40 \cdot 0$ & $68 \cdot 4$ & $0 \cdot 29142$ & $35 \cdot 8$ & $-1029 \cdot 07771$ & $67 \cdot 0$ \\
\hline $5 \mathbf{e}^{\prime}-\mathrm{O}$ & $0 \cdot 37498$ & $37 \cdot 8$ & $79 \cdot 5$ & $0 \cdot 28798$ & $33 \cdot 6$ & $-1029 \cdot 09790$ & $54 \cdot 4$ \\
\hline $5 f^{\prime}-O$ & $0 \cdot 38038$ & $41 \cdot 2$ & $67 \cdot 3$ & $0 \cdot 29221$ & $36 \cdot 3$ & $-1029 \cdot 08407$ & $63 \cdot 1$ \\
\hline $6 \mathbf{a}^{\prime}-\mathrm{O}$ & $0 \cdot 34199$ & $17 \cdot 1$ & $8 \cdot 0$ & $0 \cdot 25073$ & $10 \cdot 3$ & $-1029 \cdot 12539$ & $37 \cdot 1$ \\
\hline $6 \mathbf{b}^{\prime}-\mathrm{O}$ & $0 \cdot 34019$ & $16 \cdot 0$ & $8 \cdot 9$ & $0 \cdot 24983$ & $9 \cdot 7$ & $-1029 \cdot 14239$ & $26 \cdot 5$ \\
\hline $7 \mathbf{a}^{\prime}-\mathrm{O}$ & $0 \cdot 34608$ & $19 \cdot 7$ & $17 \cdot 8$ & $0 \cdot 25894$ & $15 \cdot 4$ & $-1029 \cdot 11375$ & $44 \cdot 4$ \\
\hline $7 b^{\prime}-O$ & $0 \cdot 33668$ & $13 \cdot 8$ & $2 \cdot 2$ & $0 \cdot 25397$ & $12 \cdot 3$ & $-1029 \cdot 15153$ & $20 \cdot 7$ \\
\hline $8 \mathbf{a}^{\prime}-\mathrm{O}$ & $0 \cdot 36099$ & $29 \cdot 0$ & $30 \cdot 3$ & $0 \cdot 27266$ & $24 \cdot 0$ & $-1029 \cdot 09714$ & $54 \cdot 8$ \\
\hline $8 \mathbf{b}^{\prime}-\mathrm{O}$ & $0 \cdot 34832$ & $21 \cdot 1$ & $5 \cdot 7$ & $0 \cdot 25971$ & $15 \cdot 9$ & $-1029 \cdot 14142$ & $27 \cdot 1$ \\
\hline $8 \mathbf{c}^{\prime}-\mathrm{O}$ & $0 \cdot 38123$ & $41 \cdot 7$ & $70 \cdot 9$ & $0 \cdot 29067$ & $35 \cdot 3$ & $-1029 \cdot 09475$ & $56 \cdot 3$ \\
\hline
\end{tabular}

range from 68 to $76 \mathrm{kcal} / \mathrm{mol}$ respectively. The $E Z$ isomer $\mathbf{8 b}-\mathrm{O}$ is favoured over the $Z$ isomer $\mathbf{8 a}-\mathrm{O}$ and the $E E$ isomer $\mathbf{8 c}-\mathrm{O}$, which may be due to lower angle strain in the $E Z$ form. Isomers [2.2.0.0], [3.0.1.0], $[3 \cdot 1 \cdot 0 \cdot 0]$, and $[4 \cdot 0 \cdot 0 \cdot 0]$ are destabilized by the presence of severe angle strain in the $-(\mathrm{CH})_{n}-$ linkages like the porphyrin isomers. In the thia-isomer [3.0.1.0], the $Z$ isomer $\mathbf{6 a}-S$ is more stable than the $E$ isomer $\mathbf{6 b}-\mathrm{S}$ by $13 \mathrm{kcal} / \mathrm{mol}$, which is the reverse when compared to the oxa and parent porphyrin isomers, and may be traced to the absence of hydrogen bonding in the case of the thiaporphyrin isomer 6b-S. In the parent porphyrin and oxaporphyrin, the $E$ isomers are stabilized by hydrogen bonding. When the inner proton repulsion is very dominating, the minimum energy structures adopt almost a bowl-like geometry and experience severe angle strain.

\subsection{Syn tautomeric form}

The relative energies and the distortion energies of the syn tautomer of porphyrin, oxaporphyrin and thiaporphyrin analogues are given in tables 4, 5 and 6 respectively. Figure 2 depicts the variation of the relative energies with porphyrin isomers, oxaporphyrin and thiaporphyrin isomers.

In this section, we discuss the syn tautomeric structure and compare the results of the parent system with that of the oxa- and thia-disubstituted analogues respectively. Like the anti tautomeric isomers, here

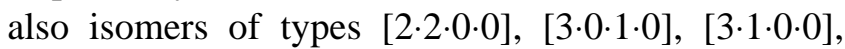
and $[4 \cdot 0 \cdot 0 \cdot 0]$ are destabilized by the presence of severe angle strain owing to the $-(\mathrm{CH})_{n}$ - linkages. All the 25 isomeric forms considered here are found to be distinct minima on the potential energy surface of the syn porphyrin isomers, a feature in slight contrast with the anti-counterparts. However, the twin isomeric forms $\mathbf{3} \mathbf{b}^{\prime}-\mathrm{X}$ and $\mathbf{3} \mathbf{c}^{\prime}$-X collapse to an intermediate bowl-like structure for the oxa- and thiaanalogues. In isomers [3.0.1.0] and [3.1.0.0], $E$ isomers $\mathbf{6} \mathbf{b}^{\prime}-\mathrm{NH}$ and $\mathbf{7} \mathbf{b}^{\prime}-\mathrm{NH}$ are more stable than the corresponding $Z$ forms $\mathbf{6} \mathbf{a}^{\prime}-\mathrm{NH}$ and $\mathbf{7} \mathbf{b}^{\prime}-\mathrm{NH}$ respectively. The $E Z$ isomer $\mathbf{8} \mathbf{b}^{\prime}-\mathrm{NH}$ is favoured over the $Z$ isomer $8 \mathbf{a}^{\prime}-\mathrm{NH}$ and $E E$ isomer $\mathbf{8 c}^{\prime}-\mathrm{NH}$, this 

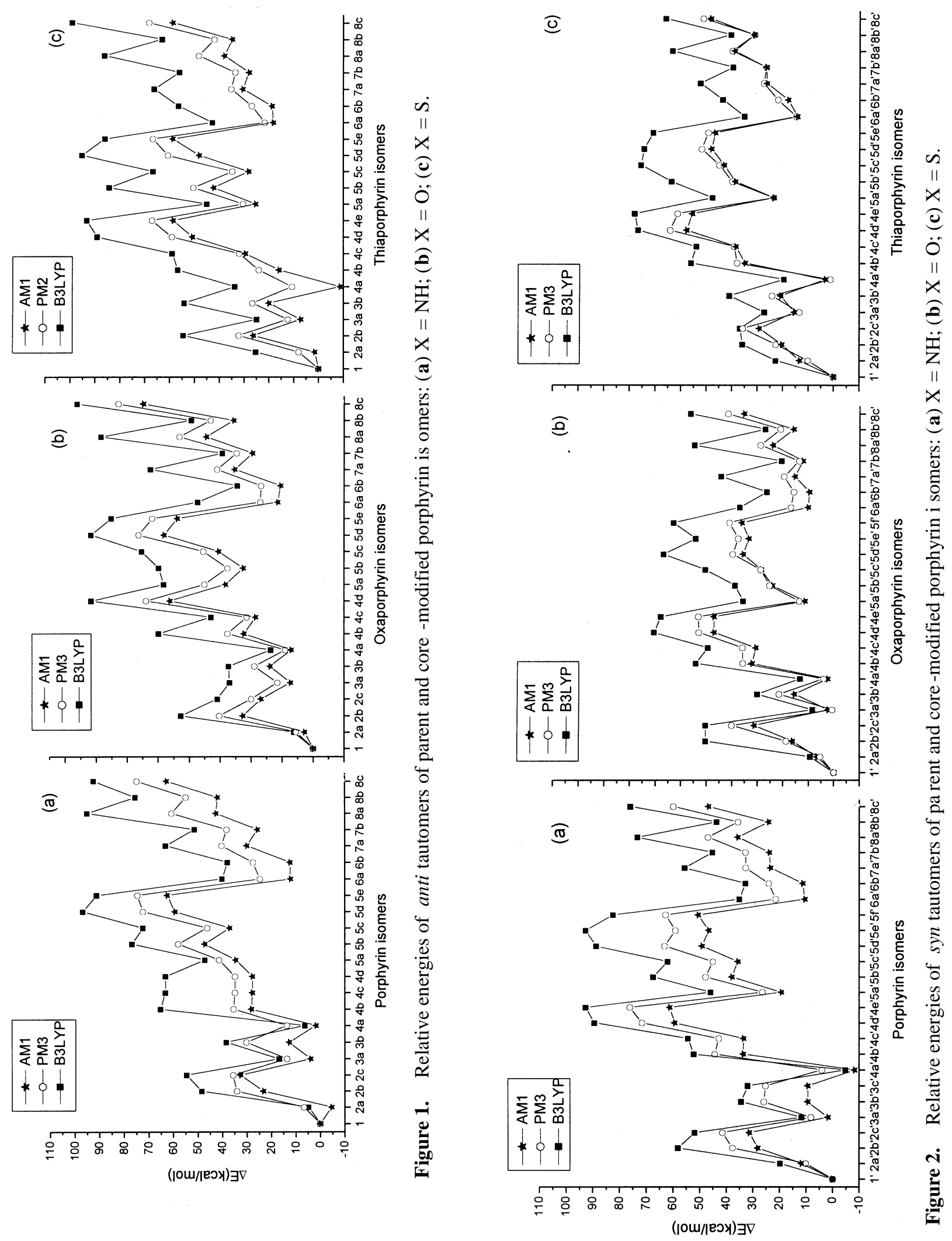
Table 6. Total energies, relative energies and distortion energies of syn tautomers of coremodified thiaporphyrin isomers obtained at semiempirical (AM1, PM3) and DFT (B3LYP/ 6-31G*//AM1) levels of theory. Total energies are in hartrees and relative energies and distortion energies (DE) are in $\mathrm{kcal} \mathrm{mol}^{-1}$.

\begin{tabular}{|c|c|c|c|c|c|c|c|}
\hline \multirow[b]{2}{*}{ Structure } & \multicolumn{3}{|c|}{ AM1 } & \multicolumn{2}{|c|}{ PM3 } & \multicolumn{2}{|c|}{ B3LYP/6-31G*//AM1 } \\
\hline & $\mathrm{HF}$ & $\Delta E$ & $\mathrm{DE}$ & $\mathrm{HF}$ & $\Delta E$ & $\mathrm{HF}$ & $\Delta E$ \\
\hline $\mathbf{1}^{\prime}-\mathrm{S}$ & 0.42352 & $0 \cdot 0$ & $10 \cdot 6$ & $0 \cdot 36831$ & $0 \cdot 0$ & $-1675 \cdot 10774$ & $0 \cdot 0$ \\
\hline $2 \mathbf{a}^{\prime}-S$ & 0.43963 & $10 \cdot 1$ & 13.9 & $0 \cdot 38971$ & $13 \cdot 4$ & $-1675 \cdot 07145$ & $22 \cdot 8$ \\
\hline $2 \mathbf{b}^{\prime}-S$ & 0.45972 & $22 \cdot 7$ & $58 \cdot 2$ & 0.40076 & $20 \cdot 4$ & $-1675 \cdot 05072$ & $35 \cdot 8$ \\
\hline $2 c^{\prime}-S$ & 0.48039 & $35 \cdot 7$ & $31 \cdot 1$ & $0 \cdot 41482$ & $29 \cdot 2$ & $-1675 \cdot 04896$ & $36 \cdot 9$ \\
\hline $3 \mathbf{a}^{\prime}-S$ & $0 \cdot 44502$ & $13 \cdot 5$ & $16 \cdot 0$ & $0 \cdot 39291$ & $15 \cdot 4$ & $-1675 \cdot 06446$ & $27 \cdot 2$ \\
\hline $3 \mathbf{b}^{\prime}-S$ & 0.46204 & $24 \cdot 2$ & $30 \cdot 5$ & 0.40147 & $20 \cdot 8$ & $-1675 \cdot 04241$ & $41 \cdot 0$ \\
\hline $4 a^{\prime}-S$ & $0 \cdot 42553$ & $1 \cdot 3$ & $15 \cdot 1$ & $0 \cdot 37349$ & $3 \cdot 2$ & $-1675 \cdot 07674$ & $19 \cdot 5$ \\
\hline $4 \mathbf{b}^{\prime}-S$ & 0.48387 & $37 \cdot 9$ & $61 \cdot 4$ & 0.42381 & $34 \cdot 8$ & $-1675 \cdot 01871$ & $55 \cdot 9$ \\
\hline $4 c^{\prime}-S$ & $0 \cdot 48587$ & $39 \cdot 1$ & $58 \cdot 8$ & 0.42953 & $38 \cdot 4$ & $-1675 \cdot 02185$ & $53 \cdot 9$ \\
\hline $4 d^{\prime}-S$ & 0.52576 & $64 \cdot 2$ & $94 \cdot 0$ & 0.46029 & $57 \cdot 7$ & -1674.98548 & $76 \cdot 7$ \\
\hline $4 e^{\prime}-S$ & 0.52125 & $61 \cdot 3$ & $92 \cdot 0$ & 0.45683 & $55 \cdot 5$ & -1674.98324 & $78 \cdot 1$ \\
\hline $5 \mathbf{a}^{\prime}-S$ & 0.46116 & $23 \cdot 6$ & $37 \cdot 6$ & 0.40599 & $23 \cdot 6$ & $-1675 \cdot 03187$ & $47 \cdot 6$ \\
\hline $5 \mathbf{b}^{\prime}-S$ & $0 \cdot 48714$ & $39 \cdot 9$ & $64 \cdot 3$ & 0.42999 & $38 \cdot 7$ & $-1675 \cdot 00620$ & $63 \cdot 7$ \\
\hline $5 c^{\prime}-S$ & 0.49529 & $45 \cdot 0$ & $84 \cdot 1$ & 0.43704 & $43 \cdot 1$ & -1674.98712 & $75 \cdot 7$ \\
\hline $5 d^{\prime}-S$ & 0.50639 & $52 \cdot 0$ & $100 \cdot 2$ & 0.44495 & $48 \cdot 1$ & -1674.98931 & $74 \cdot 5$ \\
\hline $5 e^{\prime}-S$ & $0 \cdot 50218$ & $49 \cdot 4$ & $103 \cdot 1$ & 0.44253 & $46 \cdot 6$ & -1674.99479 & $70 \cdot 9$ \\
\hline $6 \mathbf{a}^{\prime}-S$ & $0 \cdot 44706$ & $14 \cdot 8$ & $24 \cdot 2$ & $0 \cdot 39122$ & $14 \cdot 4$ & $-1675 \cdot 05159$ & $35 \cdot 2$ \\
\hline $6 \mathbf{b}^{\prime}-S$ & 0.45876 & $22 \cdot 1$ & $27 \cdot 0$ & $0 \cdot 39707$ & $18 \cdot 0$ & $-1675 \cdot 03804$ & $43 \cdot 7$ \\
\hline $7 \mathbf{a}^{\prime}-S$ & $0 \cdot 46768$ & $27 \cdot 7$ & $41 \cdot 6$ & 0.41038 & $26 \cdot 4$ & $-1675 \cdot 02402$ & $52 \cdot 5$ \\
\hline $7 b^{\prime}-S$ & $0 \cdot 46608$ & $26 \cdot 7$ & $39 \cdot 8$ & 0.41092 & $26 \cdot 7$ & $-1675 \cdot 04436$ & $39 \cdot 8$ \\
\hline $8 \mathbf{a}^{\prime}-S$ & 0.48734 & $40 \cdot 0$ & $90 \cdot 8$ & 0.43066 & $39 \cdot 1$ & $-1675 \cdot 00661$ & $63 \cdot 5$ \\
\hline $8 \mathbf{b}^{\prime}-S$ & 0.47339 & $31 \cdot 3$ & $52 \cdot 6$ & 0.41837 & $31 \cdot 4$ & $-1675 \cdot 04300$ & $40 \cdot 6$ \\
\hline $8 c^{\prime}-S$ & $0 \cdot 50562$ & $51 \cdot 5$ & $101 \cdot 8$ & 0.44561 & $48 \cdot 5$ & $-1675 \cdot 00247$ & $66 \cdot 1$ \\
\hline
\end{tabular}

may be due to the lower angle strain in the $E Z$ form $\mathbf{8} \mathbf{b}^{\prime}-\mathrm{NH}$. The distortion energy trends are also very similar in the syn-and anti-isomeric forms.

Among the syn-isomers, $\mathbf{4 a}^{\prime}-\mathrm{NH}$ (porphycene) was computed to be most stable among all the 25 isomers despite apparently significant angle strain. The intermolecular hydrogen bonding in the core seem to strongly stabilize the isomer $\mathbf{4 a}^{\prime}-\mathrm{NH}$ and importantly it is virtually planar at all levels of theory. However, among the core-modified isomers the normal $[1 \cdot 1 \cdot 1 \cdot 1]$ anti isomers was computed to be the most stable for both oxa- and thia-forms. The non-availability of the intramolecular hydrogen bonding may be the reason for such a reversal of relative energy orderings upon core-modification. Thus, hydrogen bonding in the porphyrin core is an extremely important factor in deciding the relative stabilities of the isomers. It is interesting to note that the trends in the relative energy orderings of the oxa- and thia-analogues are not drastically altered when compared to parent porphy- rin isomers (figure 2). This indicates that the coremodification is not expected to induce several structural perturbations in this class of compounds.

Figure 2 depicts the relative energy variations of the syn-isomeric forms of parent, oxa- and thia-porphyrins. While the trends obtained are similar at the three levels, the quantitative differences are substantial. It is interesting to note that AM1 values are consistently in between PM3 and B3LYP values in all cases. Consistently, the stabilities of the other porphyrin isomers are overestimated at PM3 level compared to B3LYP level. In a majority of the cases AM1 values are much closer to the PM3 values compared to the B3LYP values. Figure 1, which accounts for the relative stabilities for the anti-isomers, gives exactly the same trend. Thus, the present analysis clearly exposes the inadequacy of the semiempirical methodologies when applied to porphyrin type molecules. However, if the $a b$ initio or density functional theory based methods are intractable, 


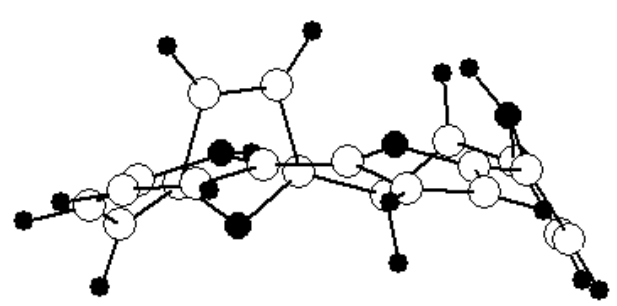

5e-NH

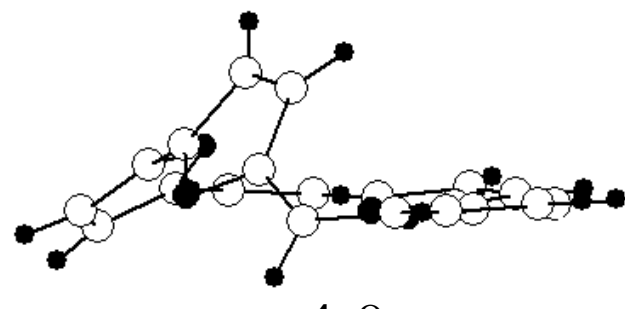

$4 c-O$

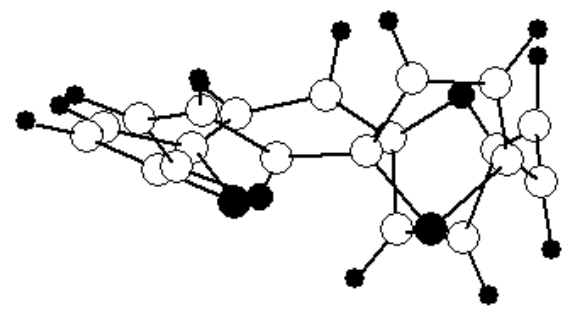

5e-S
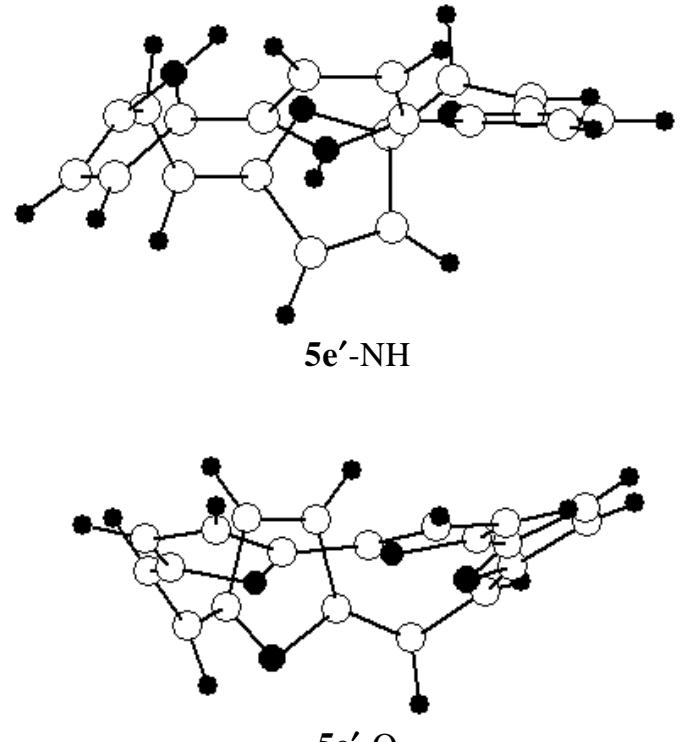

$5 \mathbf{e}^{\prime}-\mathrm{O}$

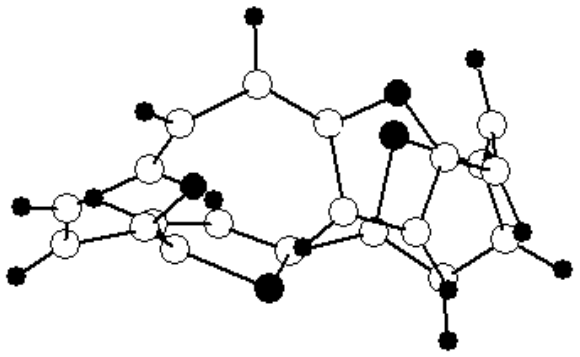

$5 \mathbf{e}^{\prime}-\mathrm{S}$

Figure 3. Representative three-dimensional structures for the highly distorted porphyrin and core-modified porphyrin isomers. The open circles and small filled circles are carbon and hydrogen. The larger circles are nitrogen, oxygen and sulphur in the corresponding isomers.

among the semiempirical methods AM1 appears to be a better choice compared to PM3 for modelling the porphyrin type systems.

\subsection{Geometrical isomerism}

Except for the parent [1.1.1.1] form, geometrical isomerism is possible for all the isomeric forms considered for the syn and anti isomers. The relative stabilities of the geometrical isomers have attracted the attention of theoreticians and experimentalists alike. In hemiporphyrin $[2 \cdot 1 \cdot 1 \cdot 0]$ and corrphycene $[2 \cdot 1 \cdot 0 \cdot 1]$ three isomers are considered in both syn and anti form (one $Z$ form and two $E$ forms). In porphycene $[2 \cdot 0 \cdot 2 \cdot 0]$ five isomers are consider (one $Z$ form, two $E Z$ forms and two $E E$ forms) in both tautomeric forms. In both syn and anti porphyrin isomers, the isomers $[2 \cdot 1 \cdot 1 \cdot 0],[2 \cdot 1 \cdot 0 \cdot 1],[2 \cdot 0 \cdot 2 \cdot 0]$, and $[2 \cdot 2 \cdot 0 \cdot 0]$ have two $-(\mathrm{CH})-$ linkages, the $Z$ isomers have lower energy compared to all other isomers in

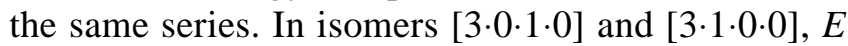
isomers are more stable than $Z$ forms in B3LYP level for the syn and anti parents and oxaporphyrin isomers. Thiaporphyrin isomers [3.0.1.0] prefer the $Z$ form over the $E$ form in both tautomeric forms. In isomer [4.0.0.0] the $E Z$ isomers are favoured over the $Z$ and $E E$ isomers, this may be due to lower angle strain in the $E Z$ form. The distortion energies for the

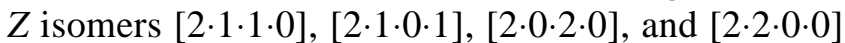
having two $-(\mathrm{CH})$ - linkages are substantially smaller compared to the $E$ isomers. Thus, the $Z$ isomers are expected to have less steric repulsion due to inner protons compared to other geometrical isomers.

In isomers isoporphycene [3.0.1.0] and [3.1.0.0], one $E$ form and one $Z$ form was considered in both syn and anti tautomers. In anti tautomeric isomers 
$[2 \cdot 1 \cdot 1 \cdot 0],[2 \cdot 1 \cdot 0 \cdot 1],[2 \cdot 0 \cdot 2 \cdot 0]$, and $[2 \cdot 2 \cdot 0 \cdot 0]$ having two $-(\mathrm{CH})$ - linkages, prefer $Z$ isomers $\mathbf{2 a}-\mathrm{X}, \mathbf{3 a}-\mathrm{X}$ in case of $[2 \cdot 1 \cdot 1 \cdot 0]$ and $[2 \cdot 1 \cdot 0 \cdot 1]$ and $Z Z$ isomers $4 \mathbf{a}-X$ and $5 \mathbf{a}-X$ in case of $[2 \cdot 0 \cdot 2 \cdot 0]$ and [2.2.0.0] where $\mathrm{X}=\mathrm{NH}, \mathrm{O}, \mathrm{S}$ respectively. This preference for the $Z$-form seems to be due to the lower steric repulsive interactions involving the inner $\mathrm{CH}$ group compared to the corresponding $E(\mathbf{2 b}-X, \mathbf{2 c}-X, \mathbf{3 b}-\mathrm{X}$ and $\mathbf{3 c}$ $X), E Z(\mathbf{4 b}-X, \mathbf{4 c}-X, \mathbf{5 b}-X$ and $\mathbf{5 c}-X)$ and $E E$ isomers $(\mathbf{4 d}-X, 4 \mathbf{e}-X, 5 d-X, 5 e-X$ and $\mathbf{5 f}-X)$. In isomers

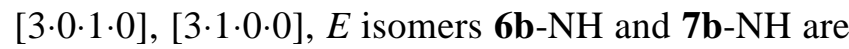
more stable than $Z$ isomers $6 \mathbf{a}-\mathrm{NH}$ and $\mathbf{7 a}-\mathrm{NH}$ in the B3LYP level. The oxa-core modified isomers seem to be following exactly similar results, and in all cases the trends observed are identical to those in the parent porphyrin isomer. However the $Z$ isomer 6a-S of $[3 \cdot 0 \cdot 1 \cdot 0]$ is more stable than the $E$ isomer 6b-S by $13 \mathrm{kcal} / \mathrm{mol}$. This is due to absence of hydrogen bonding in the case of thiaporphyrin isomers, and it is to be noted that in parent porphyrin and oxaporphyrin isomers, hydrogen bonding stabilizes the $E$ isomers. Therefore, the current study on the parent and susbstituted porphyrins exposes the severe limitations of semiempirical methods in quantitatively modelling the relative energies and out-of-plane distortion energies. However, the qualitative trends are reasonable at AM1 level of theory.

Similarly, in syn tautomers the isomers $[2 \cdot 1 \cdot 1 \cdot 0]$, $[2 \cdot 1 \cdot 0 \cdot 1],[2 \cdot 0 \cdot 2 \cdot 0],[2 \cdot 2 \cdot 0 \cdot 0]$ having $-(\mathrm{CH})_{2}-$ linkage, favours $Z$ isomers $\mathbf{2} \mathbf{a}^{\prime}-X, \mathbf{3} \mathbf{a}^{\prime}-X, \mathbf{4 a}^{\prime}-X$ and $\mathbf{5} \mathbf{a}^{\prime}$ $X$ where $X=N H, O, S$ and this preference may be due to the less steric interactions involving the inner $\mathrm{CH}$ group. However, in case of isomers of types [3.1.0.0] and [3.0.1.0], $E$ isomer $\mathbf{6} \mathbf{b}-\mathrm{NH}$ and $7 \mathbf{b}-\mathrm{NH}$ is more stable than $Z$ isomer $\mathbf{6 a - N H}$ and $\mathbf{7 a}-\mathrm{NH}$ in the B3LYP level. Oxaporphyrin isomers follow similar results. As has been observed for the thiaisomers the lack of hydrogen bonding possibility leads to the stabilization of $Z$ isomer $\mathbf{6 a}^{\prime}-S$ than the $E$ isomer $\mathbf{6} \mathbf{b}^{\prime}-\mathrm{S}$ by $8 \mathrm{kcal} / \mathrm{mol}$. Isomer [4.0.0.0] favoured $E Z$ isomer 8b-NH than $Z$ isomer 8a-NH and $E E$ isomer $8 \mathbf{c}-\mathrm{NH}$. Similar trend was observed in oxa- and thia-porphyrin isomers having [4.0.0.0] connectivity.

\section{Conclusions}

The present study reports the results of semiempirical (AM1 and PM3) and density functional calculations (B3LYP/6-31G*) on a series of syn and anti tautomeric forms of porphyrin isomers and their oxa- and thia-analogues. Several factors, such as angle strain, inner proton repulsion, length of the bridge, and the type of geometric isomerism, $E$ or $Z$, play vital roles in deciding the relative stability ordering of the porphyrin isomers. The present computational study exposes the limitations of the semiempirical theoretical methodologies when applied to porphyrin-type molecules. Between the two semiempirical methods employed, AM1 performs consistently better than PM3 in reproducing the relative energy values and orderings. The trends in the relative energy orderings for the parent porphyrins are not drastically altered when compared to the oxa- and thia-analogues in both the syn and anti tauomers.

\section{Acknowledgement}

Dr J S Yadav is thanked for support and encouragement.

\section{References}

1. Lecomte C, Rohmer M-M and Benard M 2000 The porphyrin handbook (eds) K M Kadish, K M Smith and R Guilard vol. 2, pp. 39-78; Shelnutt J A 2000 The porphyrin handbook (eds) K M Kadish, K M Smith and R Guilard vol. 7, pp. 167-223

2. Ghosh A 2000 The porphyrin handbook (eds) K M Kadish, K M Smith and R Guilard vol. 7, pp. 1-38; Jasat A and Dolphin D 1997 Chem. Rev. 97 2267

3. Stilts C E, Nelen M I, Hilmey D G, Davies S R, Gollnick S O, Oseroff A R, Gibson S L, Hilf R and Detty M R 2000 J. Med. Chem. 432403

4. Battersby A R 2000 Nat. Prod. Rep. 17507

5. Furuta H, Kubo N, Maeda H, Ishizuka T, Osuka A, Nanami $H$ and Ogawa T 2000 Inorg. Chem. 39 5424; Harmjanz M, Gill H S and Scott M J $2000 \mathrm{~J}$. Am. Chem. Soc. 122 10476; Anzenbacher P Jr, Jursikova K and Sessler J L 2000 J. Am. Chem. Soc. 122 9350; Gisselbrecht J P, Gross M, Vogel E and Sessler J L 2000 Inorg. Chem. 39 2850; Anand V G, Pushpan S K, Venkatraman S, Narayanan S J, Dey A, Chandrashekar T K, Roy R, Joshi B S, Deepa S and Sastry G N 2002 J. Org. Chem. 676309

6. Ravikumar M and Chandrashekar T K 1999 J. Inc. Phen Macro Chem. 35 553; Zenkevich E, Sagun E, Knyukshto V, Shulga A, Mironov A, Efremova O, Bonnett R, Songca S P and Kassem M 1996 J. Photochem. Photobiol. B33 171

7. Ghosh A 1996 Acc. Chem. Res. 31189

8. Vogel E, Kocher M, Schmickler H and Lex J 1986 Angew. Chem., Int. Ed. Engl. 25 257; Furukta H, Maeda H and Osuka A 2000 J. Org. Chem. 65 4222; Furukta H, Maeda H and Osuka A $2000 \mathrm{~J}$. Am. Chem. Soc. 122 803; Chmielelwski P J, Latos-Grazynski L, 
Rachlewicz K and Glowiak T 1994 Angew. Chem., Int. Ed. Engl. 33779

9. Wu Y-D, Chan K W K, Yip C-P, Vogel E, Plattner D A and Houk K N 1997 J. Org. Chem. 629240

10. Ghosh A and Jynge K 1997 J. Phys. Chem. B101 5459

11. Sessler J L, Brucker E A, Weghorn S J, Kisters M, Schafer M, Lex J and Vogel E 1994 Angew. Chem., Int. Ed. Engl. 332308

12. Vogel E, Broring M, Erben C, Demuth R, Lex J, Nendel M and Houk K N 1997 Angew. Chem., Int.
Ed. Engl. 36 353; Szterenberg L and Latos-Grazynski L 1997 Inorg. Chem. 366287

13. Punnagai M and Sastry G N 2004 J. Mol. Struc. (Theochem.) (accepted)

14. Dewar M J S, Zoebisch Z, Healy E F and Stewart J J P 1985 J. Am. Chem. Soc. 1073902

15. Stewart J J P 1989 J. Comput. Chem. 10 209; Stewart J J P 1989 J. Comput. Chem. 10221

16. Gaussian '98 2001 Revision A.11.2, Frisch M J et al Gaussian Inc, Pittsburgh PA 